\title{
WHICH SEQUENCES OF HOLES ARE ADMISSIBLE FOR PERIODIC HOMOGENIZATION WITH NEUMANN BOUNDARY CONDITION?
}

\author{
Alain Damlamian ${ }^{1}$ and Patrizia Donato ${ }^{2,3}$
}

\begin{abstract}
In this paper we give a general presentation of the homogenization of Neumann type problems in periodically perforated domains, including the case where the shape of the reference hole varies with the size of the period (in the spirit of the construction of self-similar fractals). We shows that $H^{0}$-convergence holds under the extra assumption that there exists a bounded sequence of extension operators for the reference holes. The general class of Jones-domains gives an example where this result applies. When this assumption fails, another approach, using the Poincaré-Wirtinger inequality is presented. A corresponding class where it applies is that of John-domains, for which the PoincaréWirtinger constant is controlled. The relationship between these two kinds of assumptions is also clarified.
\end{abstract}

Mathematics Subject Classification. 35B27, 35J25, 46E35.

Received December 17, 2001.

Dedicated to the memory of Jacques-Louis Lions (1928-2001)

\section{INTRODUCTION}

The aim of this paper is to give a general presentation of the homogenization of Neumann type problems in a periodically perforated domain $\Omega_{\varepsilon}=\Omega \backslash T_{\varepsilon}$ obtained by removing a compact set $T_{\varepsilon}$ of holes from a given domain $\Omega$ of $\mathbb{R}^{N}$.

The holes $T_{\varepsilon}$ are $\varepsilon$-periodically distributed and $\varepsilon$-homothetic to a reference hole $\mathcal{T}_{\varepsilon}$, the shape of which can also vary with $\varepsilon$ approaching, for instance, a self-similar fractal.

Throughout this paper, $\varepsilon$ will denote the general term of a sequence of positive reals which converges to zero and we will assume that the characteristic function of $\mathcal{T}_{\varepsilon}$ converges to that of a limit set $\mathcal{T}_{0}$ almost everywhere.

We consider the following type of problems

$$
\left\{\begin{array}{l}
-\operatorname{div}\left(A^{\varepsilon} \nabla u_{\varepsilon}\right)=f \quad \text { in } \Omega_{\varepsilon} \\
\left(A^{\varepsilon}(x) \nabla u_{\varepsilon}\right) \cdot \nu=0 \quad \text { on } \partial T_{\varepsilon} \\
u_{\varepsilon}=0 \quad \text { on } \partial \Omega
\end{array}\right.
$$

Keywords and phrases: Periodic homogenization, perforated domains, $H^{0}$-convergence, Poincaré-Wirtinger inequality, Jones domains, John domains.

${ }^{1}$ Laboratoire d'Analyse et de Mathématiques Appliquée, UMR 8050 du CNRS, Universités de Marne-la-Vallée et Paris 12 Val-de-Marne, Université Paris 12, 94010 Créteil Cedex France; e-mail: damlamian@univ-paris12.fr

${ }^{2}$ Laboratoire de Mathématiques Raphaël Salem, Université de Rouen, Site Colbert, 76821 Mont-Saint-Aignan Cedex, France; e-mail: donato@univ-rouen.fr

${ }^{3}$ Université Paris VI, Laboratoire Jacques-Louis Lions, Boîte Courrier 187, 75252 Paris Cedex, France;

e-mail: donato@ann.jussieu.fr 
where $f$ is given in $L^{2}(\Omega),\left(A^{\varepsilon}\right)$ is a sequence of uniformly bounded and uniformly coercive matrix-valued functions, $\Omega_{\varepsilon}=\Omega \backslash T_{\varepsilon}$ of the form

$$
T_{\varepsilon}=\bigcup\left\{\varepsilon\left(k+\mathcal{T}_{\varepsilon}\right) ; k \in \mathbb{Z}^{N}, \varepsilon\left(k+\mathcal{T}_{\varepsilon}\right) \subset \Omega\right\}
$$

and $\nu$ is the outward normal unit vector on the boundary of $\Omega_{\varepsilon}$. The solution $u_{\varepsilon}$ belongs to the Hilbert space

$$
\mathcal{V}_{\varepsilon}=\left\{v \in H^{1}\left(\Omega_{\varepsilon}\right): v_{\mid \partial \Omega}=0\right\},
$$

equipped with the $H^{1}$-norm. Here $H^{1}(\mathcal{O})$ for a domain $\mathcal{O}$ is the usual Sobolev space of functions in $L^{2}(\mathcal{O})$ with distributional first derivatives also in $L^{2}(\mathcal{O})$.

Homogenization (without holes) goes back to the late 1960's. We refer to the by now classical well-known works of Spagnolo [28], Bensoussan et al. [5] or Sanchez-Palencia [26].

Homogenization in perforated domains has been widely studied starting in the late 1970's. The first papers on the subject (Cioranescu and Saint Jean Paulin [12]), in the case of a fixed reference hole, made use of the existence of an extension operator $P_{\varepsilon}$ from $\mathcal{V}_{\varepsilon}$ to $H_{0}^{1}(\Omega)$ such that for some positive constant $c$ independent of $\varepsilon$,

$$
\left\|\nabla\left(P_{\varepsilon} v\right)\right\|_{\left(L^{2}(\Omega)\right)^{N}} \leq c\|\nabla v\|_{\left(L^{2}\left(\Omega_{\varepsilon}\right)\right)^{N}}, \quad \forall v \in \mathcal{V}_{\varepsilon} .
$$

In a recent paper [8], this situation was formalized in the notion of $H^{0}$-convergence which we recall below. It is an extension to perforated domains of the $H$-convergence introduced in 1977 by Murat and Tartar in [24] and [30] (see also [25]).

\section{Notations.}

- $M(\alpha, \beta, \Omega)$ denotes, for two positive reals $\alpha<\beta$, the set of the $N \times N$ matrix-valued functions $A$ defined on $\Omega$ and satisfying

$$
\left\{\begin{array}{l}
A \text { measurable on } \Omega, \\
(A(x) \lambda, \lambda) \geq \alpha|\lambda|^{2}, \quad(A(x) \lambda, \lambda) \geq \beta^{-1}|A(x) \lambda|^{2} \quad \forall \lambda \in \mathbb{R}^{N}, \text { a.e. } x \in \Omega ;
\end{array}\right.
$$

- $\chi_{E}$ denotes the characteristic function of a subset $E$ of $\mathbb{R}^{N}$;

- $|E|$ denotes the Lebesgue measure of a Lebesgue-measurable subset $E$ of $\mathbb{R}^{N}$;

- $\tilde{v}\left(\right.$ or $\left.[v]^{\sim}\right)$ denotes the zero extension on $\Omega$ of any vector function $v$ defined on $\Omega_{\varepsilon}$;

- $\nu$ denotes the unitary external normal vector with respect to $\Omega_{\varepsilon}$;

- $\mathcal{M}_{E}(v)=\frac{1}{[E]} \int_{E} v(x) \mathrm{d} x$ for every Lebesgue-measurable subset of $\mathbb{R}^{N}$ with $\left.\mid E\right]>0$.

Definition $1.1[8]$. The sequence $\left\{T_{\varepsilon}\right\}_{\varepsilon}$ of compacts subsets of $\Omega$ is said to be admissible (in $\Omega$ ) if

i) every $L^{\infty}$ weak $*$-limit point of $\left\{\chi_{\Omega_{\varepsilon}}\right\}_{\varepsilon}$ is positive a.e. in $\Omega$

ii) there exist a positive real $c$, independent of $\varepsilon$, and a sequence $\left\{P_{\varepsilon}\right\}_{\varepsilon}$ of linear extension-operators such that for each $\varepsilon$

$$
\left\{\begin{array}{l}
P_{\varepsilon} \in \mathcal{L}\left(\mathcal{V}_{\varepsilon}, H_{0}^{1}(\Omega)\right) \\
\left(P_{\varepsilon} v\right)_{\left.\right|_{\varepsilon}}=v \quad \forall v \in \mathcal{V}_{\varepsilon}, \\
\left\|\nabla\left(P_{\varepsilon} v\right)\right\|_{\left(L^{2}(\Omega)\right)^{N}} \leq c\|\nabla v\|_{\left(L^{2}\left(\Omega_{\varepsilon}\right)\right)^{N}} \quad \forall v \in \mathcal{V}_{\varepsilon} .
\end{array}\right.
$$

Observe that by (1.5), the Poincaré and Sobolev inequalities hold in $\mathcal{V}_{\varepsilon}$ with a constant independent of $\varepsilon$.

Definition 1.2 [8]. Let $\left\{A^{\varepsilon}\right\}_{\varepsilon}$ in $M(\alpha, \beta, \Omega),\left\{T_{\varepsilon}\right\}_{\varepsilon}$ be admissible in $\Omega$ and for every $\varepsilon$, denote the adjoint operator of $P_{\varepsilon}$ by $P_{\varepsilon}^{*}$. 
The sequence $\left\{\left(A^{\varepsilon}, T_{\varepsilon}\right)\right\}_{\varepsilon}$ is said to $H^{0}$-converge to the matrix $A^{0}$ of $M\left(\alpha^{\prime}, \beta^{\prime}, \Omega\right)$ (and denoted $\left.\left(A^{\varepsilon}, T_{\varepsilon}\right) \stackrel{H^{0}}{\longrightarrow} A^{0}\right)$ if and only if, for every function $g$ in $H^{-1}(\Omega)$, the solution $v_{\varepsilon}$ of

$$
\left\{\begin{array}{l}
-\operatorname{div}\left(A^{\varepsilon} \nabla v_{\varepsilon}\right)=P_{\varepsilon}^{*} g \quad \text { in } \Omega_{\varepsilon} \\
\left(A^{\varepsilon} \nabla v_{\varepsilon}\right) \cdot \nu=0 \quad \text { on } \partial T_{\varepsilon} \\
v_{\varepsilon}=0 \quad \text { on } \partial \Omega
\end{array}\right.
$$

satisfies the weak convergences

$$
\begin{gathered}
P_{\varepsilon} v_{\varepsilon} \rightarrow v \quad \text { weakly in } H_{0}^{1}(\Omega), \\
A^{\varepsilon} \widetilde{\nabla v_{\varepsilon}} \rightarrow A^{0} \nabla v \quad \text { weakly in }\left(L^{2}(\Omega)\right)^{N},
\end{gathered}
$$

where $v$ is the unique solution of the following problem:

$$
\left\{\begin{array}{l}
-\operatorname{div}\left(A^{0} \nabla v\right)=g \text { in } \Omega \\
v=0 \quad \text { on } \partial \Omega
\end{array}\right.
$$

Remark 1.3. Suppose that in (1.4) the whole sequence $\chi_{\Omega_{\varepsilon}}$ converges to some function $\theta$. Then, in order to have $\left(A^{\varepsilon}, T_{\varepsilon}\right) \stackrel{H^{0}}{\longrightarrow} A^{0}$ it is enough to check (1.7) and (1.8) when the right-hand side in (1.6) is $f \in L^{2}(\Omega)$ and the right-hand side in (1.9) is replaced by $\theta f$ (see [8]).

The definition of $H^{0}$-convergence is independent of the sequence $\left\{P_{\varepsilon}\right\}_{\varepsilon}$ ([8], Prop. 2.7). Moreover the following compactness result holds:

Theorem 1.4 [8]. Let $\left\{T_{\varepsilon}\right\}_{\varepsilon}$ be an admissible sequence in $\Omega$ and $\left\{A^{\varepsilon}\right\}_{\varepsilon}$ be in $M(\alpha, \beta, \Omega)$. Then, there exist a subsequence (still denoted $\{\varepsilon\}$ ) and a matrix $A^{0}$ in $M\left(\frac{\alpha}{c_{1}^{2}}, \beta, \Omega\right)$, with c given in (1.5), such that $\left\{\left(A^{\varepsilon}, T_{\varepsilon}\right)\right\}_{\varepsilon}$ $H^{0}$-converges to $A^{0}$.

The question here (as it is for the usual $H$-convergence) is whether the whole sequence converges and if so, to what limit. Sections 2-4 of this paper present results concerning a general class of sequences of holes, for which convergence holds. They generalize the classical periodic case (see [12] and [13]), where $A^{\varepsilon}$ is of the form

$$
A^{\varepsilon}(x)=A\left(\frac{x}{\varepsilon}\right) \quad \text { a.e. in } \mathbb{R}^{N},
$$

with $A(y)=\left(a_{i j}(y)\right)_{i j}$, defined on $\mathbb{R}^{N}$, is such that

$$
\left\{\begin{array}{c}
A \quad Y-\text { periodic } \\
A \in M(\alpha, \beta, Y)
\end{array}\right.
$$

and $Y=\left[0, l_{1}\left[\times . . \times\left[0, l_{N}\left[\right.\right.\right.\right.$. Furthermore, $T_{\varepsilon}$ is a finite union of holes

$$
T_{\varepsilon}=\bigcup\left\{\varepsilon\left(k_{l}+\mathcal{T}\right) ; k \in \mathbb{Z}^{N}, \varepsilon(k+\mathcal{T}) \subset \Omega\right\},
$$

$\mathcal{T} \subset Y$ being a reference hole satisfying some suitable assumptions in $\bar{Y}$. In this case there exists a constant matrix $A^{0}$, explicitly computable (see Rem. 2.11), such that $\left(A^{\varepsilon}, T_{\varepsilon}\right) \stackrel{H^{0}}{\longrightarrow} A^{0}$. Recall that in this situation, the $\theta$ of Remark 1.3 is given by $\theta=\frac{|Y \backslash \mathcal{T}|}{|Y|}$. 
The fact that $A^{0}$ is independent of $x$ is a simple consequence of the $\varepsilon$-periodicity of the problem for each $\varepsilon$. Indeed, any time the coefficients and the characteristic function of the perforated domain are $\varepsilon$-periodic, even if they are not rescaled from a fixed $\mathscr{Z}$-periodic function, provided $H^{0}$-convergence holds for a sequence.

Theorem 2.10 shows that $H^{0}$-convergence holds for the whole sequence, if the holes are defined by (1.2), under the extra assumption that there exists a bounded sequence of extension operators from $H^{1}\left(Y \backslash \mathcal{T}_{\varepsilon}\right)$ to $H^{1}(Y)$. The reference cell $Y$ can be quite general, provided it has the paving property (Def. 2.3).

In Section 4 we show how the sequences $\left\{\mathcal{T}_{\varepsilon}\right\}_{\varepsilon}$ can be chosen in the general class of Jones-domains (Def. 4.3), an example of which is the two-dimensional snowflake (Cor. 4.6).

An alternate approach in the periodic case with a fixed reference hole, has been to prove the following two convergences

$$
\begin{cases}i) & \widetilde{u}_{\varepsilon} \rightarrow \theta u \quad \text { weakly in } L^{2}(\Omega), \\ i i) \quad\left\|u_{\varepsilon}-u\right\|_{L^{2}\left(\Omega_{\varepsilon}\right)} \rightarrow 0\end{cases}
$$

where $u$ is the solution of problem (1.9) with right-hand side $f \in L^{2}(\Omega)$ and $\theta=\frac{|Y \backslash T|}{|Y|}$ (see Hruslov [18], Allaire-Murat [2] and Briane [7]). One can observe that convergence (1.7) implies convergences (1.13).

For example, in [2] (in the same geometrical setup as in [12] with a somewhat different conditions on the reference hole), the authors introduce the sequence $\left\{\bar{u}_{\varepsilon}\right\}_{\varepsilon}$ of the local averages (on each $\varepsilon$-sized cell) and prove that the sequence $\left\{\bar{u}_{\varepsilon}\right\}_{\varepsilon}$ satisfies the Kolmogorov criterion for the strong compactness in $L^{2}(\Omega)$. This yields convergences (1.13). The main ingredient is the Poincaré-Wirtinger inequality in $Y \backslash T$ and the fact that $\widetilde{\nabla u_{\varepsilon}}$ is bounded in $L^{2}(\Omega)$. Convergence (1.8) can also be shown, by similar arguments.

In [7], this approach is successfully applied to a particular situation of a sequence of reference holes ("small bridges") where there are no uniform extension operators (satisfying (1.4)) but where the Poincaré-Wirtinger constant is controlled.

In Section 5-7 we present a general class for which this holds. In Theorem 5.10 we state the main convergence result. In Section 7 we show how the sequences $\left\{\mathcal{T}_{\varepsilon}\right\}_{\varepsilon}$ can be chosen in the more general class of John-domains (Def. 7.4), for which the Poincaré-Wirtinger constant is controlled (but for which an extension operator may not even exist).

Finally, let us mention that another approach to these type of problems is presented in several papers of Zhikov (see [33]), where the notion of " $p$-connectedness" is introduced and studied (see Rem. 6.5).

$$
\text { Plan: }
$$

2. $H^{0}$ convergence in the periodic case;

3. Poincaré-Wirtinger. inequalities and proof of Theorem 2.10;

4. domains for which the extension property holds;

5. the case without extension property;

6. proof of the results of Section 5;

7. domains for which the Poincaré-Wirtinger property holds.

\section{2. $H^{0}$-CONVERGENCE IN THE PERIODIC CASE}

We start this section by describing the geometric setting.

Definition 2.1. A closed set $T_{\varepsilon}\left(\mathbb{R}^{N}, \mathcal{S}\right)$ is called a closed $\varepsilon$-periodic array whenever there exists a compact set $\mathcal{S}$ of $\mathbb{R}^{N}$ and a basis $\left(b_{1}, \ldots, b_{N}\right)$ (not necessarily orthonormal) such that

$$
T_{\varepsilon}\left(\mathbb{R}^{N}, \mathcal{S}\right)=\bigcup_{k \in \mathbb{Z}^{N}} \varepsilon\left(\mathcal{S}+\sum_{l=1}^{N} k_{l} b_{l}\right)
$$


For a given bounded domain $\Omega$ of $\mathbb{R}^{N}$ we set

$$
T_{\varepsilon}(\Omega, \mathcal{S})=\left\{\bigcup \varepsilon\left(\mathcal{S}+\sum_{l=1}^{N} k_{l} b_{l}\right), k \in \mathbb{Z}^{N}, \varepsilon\left(\mathcal{S}+\sum_{l=1}^{N} k_{l} b_{l}\right) \subset \Omega\right\}
$$

Remark 2.2. In practice, what is given is a closed $\varepsilon$-periodic array and the question is to represent it under the form (2.1). Clearly, both the set $S$ and the basis $\left(b_{1}, \ldots, b_{N}\right)$ are not uniquely defined. For example, for the same $S$, another basis satisfies also (2.1) if and only if the matrix of the change of basis has only integer entries and its determinant equals \pm 1 .

Definition 2.3. A connected open set $\mathscr{H}$ of $\mathbb{R}^{N}$ has the paving property with respect to the basis $\left(b_{1}, \ldots, b_{N}\right)$ if and only if

$$
\mathbb{R}^{N}=\bigcup_{k \in \mathbb{Z}^{N}} \overline{\mathscr{H}^{k}}, \quad \mathscr{H}^{k}=\mathscr{H}+\sum_{l=1}^{N} k_{l} b_{l}
$$

with $k=\left(k_{1}, \ldots, k_{N}\right)$ and $\mathscr{Y}^{k} \cap \mathscr{Y}^{h}=\emptyset$ for all $k, h \in \mathbb{Z}^{N}, k \neq h$.

Remark 2.4. Definition 2.3 is a particular case of the general geometric notion of fundamental domain under the action of a group.

The canonical projection $\Pi$ of $\overline{\mathscr{H}}$ into the periodic torus associated with the basis $\left(b_{1}, \ldots, b_{N}\right)$ of $\mathbb{R}^{N}$ is actually onto. Consequently, functions defined on $R^{N}$ which admit $\left(b_{1}, \ldots, b_{N}\right)$ as periods, can be seen as periodic fonctions on $\overline{\mathscr{H}}$. For simplicity, we will say that such fonctions are $\mathscr{\mathscr { J }}$-periodic. In particular, we denote by $H_{\text {per }}^{1}(\mathscr{Y})$ the space of $\mathscr{H}$-periodic functions in $H_{\text {loc }}^{1}\left(\mathbb{R}^{N}\right)$. Similarly, if $\mathcal{S}$ is a compact subset of $\mathscr{Y}$, we denote by $H_{\text {per }}^{1}(\mathscr{Y} \backslash \mathcal{S})$ the space of $\mathscr{Y}$-periodic functions in $H^{1}(\mathscr{H} \backslash \mathcal{S})$.

As in $(1.10,1.11)$, let $A^{\varepsilon}$ be given by

$$
A^{\varepsilon}(x)=A\left(\frac{x}{\varepsilon}\right) \quad \text { a.e. in } \mathbb{R}^{N}
$$

where $A(y)=\left(a_{i j}(y)\right)_{i j}$ defined on $\mathbb{R}^{N}$ is such that

$$
\left\{\begin{array}{l}
A \quad \mathscr{H}-\text { periodic } \\
A \in M(\alpha, \beta, \mathscr{Y})
\end{array}\right.
$$

In the present section, we will make the following:

Assumption 2.5. A basis $\left(b_{1}, \ldots, b_{N}\right)$ is given in $\mathbb{R}^{N}$. We assume that $\left(\mathcal{T}_{\varepsilon}\right)_{\varepsilon}$ is a sequence of compact sets of $\mathbb{R}^{N}$, such that there exists a connected open set $\mathscr{H}$, with piece-wise smooth boundary, having the paving property with respect to the basis $\left(b_{1}, \ldots, b_{N}\right)$ and

$$
\text { for every } \varepsilon>0, \quad \mathcal{T}_{\varepsilon} \subset \mathscr{Y} \text { and } \mathscr{Y} \varepsilon \doteq \mathscr{Y} \backslash \mathcal{T}_{\varepsilon} \text { is connected. }
$$

Remark 2.6. i) If all the $\mathcal{T}_{\varepsilon}$ 's are contained in a fixed compact subset of $\mathscr{Y}$, the smoothness assumption on the boundary of $\mathscr{Z}$ it is not restrictive. Indeed, one can modify $\mathscr{Z}$ in order to have a piece-wise $C^{\infty}$ boundary (even piece-wise affine).

ii) Assumption 2.5 implies that $\mathbb{R}^{N} \backslash T_{\varepsilon}\left(\mathbb{R}^{N}, \mathcal{T}_{\varepsilon}\right)$ is connected. It also implies that (but is strictly stronger than) the fact that $\Pi(\overline{\mathscr{Y}} \varepsilon)$ is connected in the torus (as well as the image of $\mathbb{R}^{N} \backslash T_{\varepsilon}\left(\mathbb{R}^{N}, \mathcal{T}_{\varepsilon}\right)$ in the corresponding $\varepsilon$-torus).

Exemples 2.7. Observe that for a given sequences of compact $\varepsilon$-periodic arrays, the choice of a $\mathscr{H}$ verifying Assumption 2.5 is not always straightforward. 

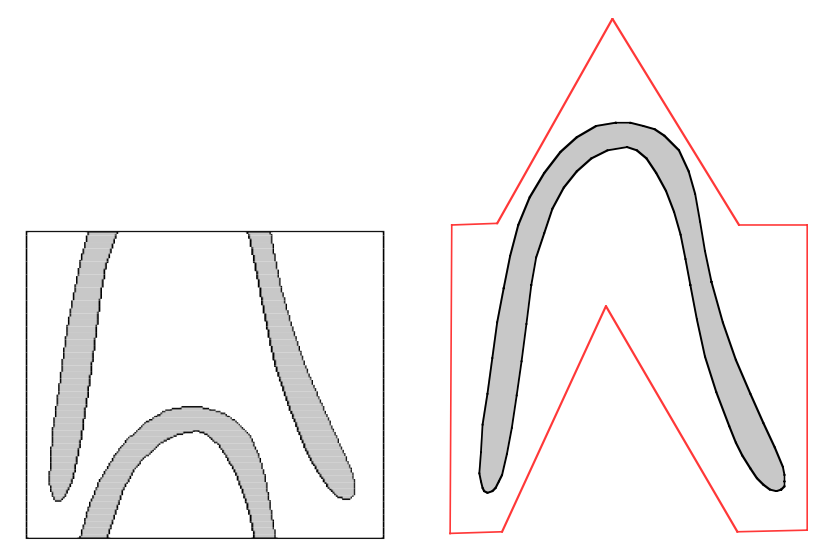

FIGURE 1
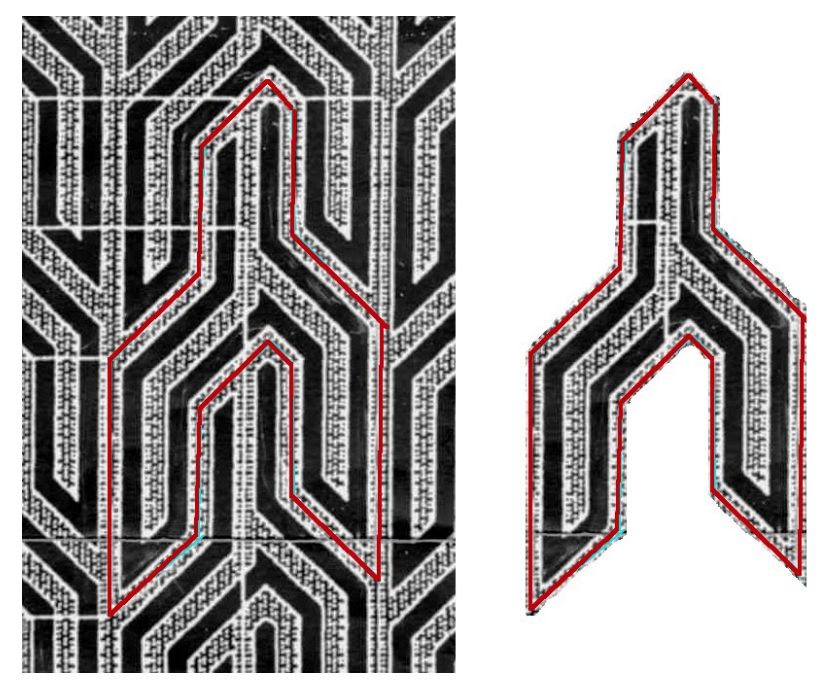

Figure 2

Figure 1 shows a situation in $\mathbb{R}^{2}$ for which one cannot choose $\mathscr{H}$ as a parallelepipedon and gives a possible choice of $\mathscr{Y}$ in order to satisfy Assumption 2.5.

Another example, in two dimensions, can be found in Acerbi et al. ([1], Sect. 2), where it is pointed out that no rectangle $\mathscr{Z}$ satisfy Assumption 2.5. Actually, it suffices to choose the open set $\mathscr{Y}$ as in Figure 2 in order to satisfy this assumption.

On the other hand, for the cases of Figures 3, no $\mathscr{Y}$ exists for which Assumption 2.5 holds.

In fact, in both cases, $\mathbb{R}^{N} \backslash T_{\varepsilon}\left(\mathbb{R}^{N}, \mathcal{T}_{\varepsilon}\right)$ is not connected. Observe, however, that in the first case of Figure 3, the image of $\mathbb{R}^{N} \backslash T_{\varepsilon}\left(\mathbb{R}^{N}, \mathcal{T}_{\varepsilon}\right)$ in the $\varepsilon$-torus is connected. This is not true for the second one.

For a given bounded domain $\Omega$ of $\mathbb{R}^{N}$ and a given sequence of closed $\varepsilon$-periodic arrays $T_{\varepsilon}\left(\mathbb{R}^{N}\right)$, we set

$$
\Omega_{\varepsilon}=\Omega \backslash T_{\varepsilon}\left(\Omega, \mathcal{T}_{\varepsilon}\right)
$$

where $T_{\varepsilon}\left(\Omega, \mathcal{T}_{\varepsilon}\right)$ is given by Definition 2.1. When there is no ambiguity, we will simply use $T_{\varepsilon}$ instead of $T_{\varepsilon}\left(\Omega, \mathcal{T}_{\varepsilon}\right)$. 

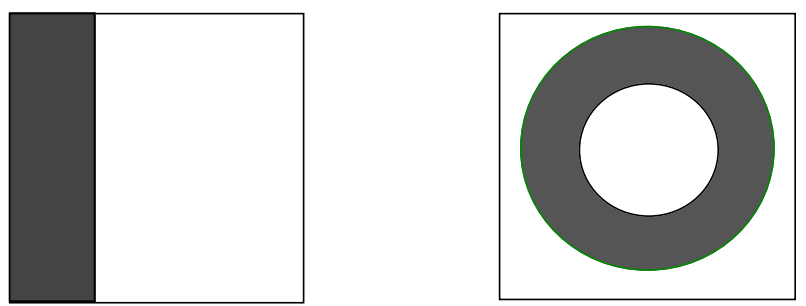

Figure 3

In this situation, a sequence of extension operators $\left\{P_{\varepsilon}\right\}_{\varepsilon}$ satisfying (1.5) is easily constructed from a similar sequence in the reference cell as shown in the following proposition. The proof follows immediately by a mere change of scale:

Proposition 2.8. Under Assumption 2.5, let $\Omega_{\varepsilon}$ be defined by (2.6). Suppose that for every $\varepsilon$ there exists an extension operator $\mathscr{P}_{\varepsilon}$ from $H^{1}\left(\mathscr{H}_{\varepsilon}\right)$ to $H^{1}(\mathscr{Y})$ having the following properties for some positive number $c_{1}$ :

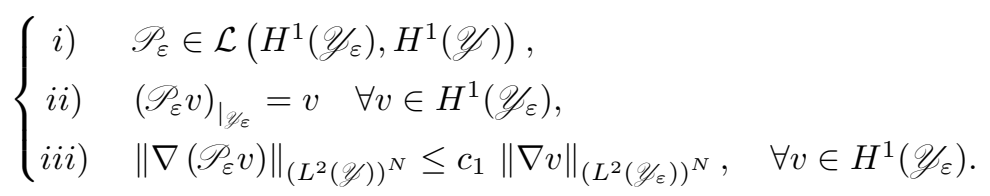

Then there exists a sequence $\left\{P_{\varepsilon}\right\}_{\varepsilon}$ of linear extension-operators satisfying (1.5).

Thanks to Theorem 1.4, we have the following trivial corollary:

Corollary 2.9. Under the assumptions of Proposition 2.8 and (1.4),

i) the sequence $\left\{T_{\varepsilon}\right\}_{\varepsilon}$ is admissible in $\Omega$;

ii) for any sequence $\left\{A^{\varepsilon}\right\}_{\varepsilon}$ defined by (2.4, 2.5) there exists a subsequence subsequence $\left\{\varepsilon^{\prime}\right\}$ such that $\left\{\left(A^{\varepsilon^{\prime}}, T_{\varepsilon^{\prime}}\right)\right\}_{\varepsilon^{\prime}} H^{0}$-converges.

It remains to characterize all the possible $H^{0}$-limits. In this direction, we now state Theorem 2.10 below.

Theorem 2.10. Under Assumption 2.5, let $\Omega_{\varepsilon}$ be defined by (2.6). Suppose that for every $\varepsilon$, there exists a linear extension operator $\mathcal{Q}_{\varepsilon}$ from $H^{1}(\mathscr{Y} \varepsilon)$ to $H^{1}(\mathscr{Y})$ satisfying

$$
\forall \varepsilon, \forall v \in H^{1}(\mathscr{Y} \varepsilon), \quad\left\|\mathcal{Q}_{\varepsilon} v\right\|_{H^{1}(\mathscr{Y})} \leq c_{0}\|v\|_{H^{1}(\mathscr{Y} \varepsilon)},
$$

for some positive $c_{0}$. Suppose furthermore that there exists a compact set $\mathcal{T}_{0}$ in $\mathscr{Y}$ for which, $\mathscr{Y} 0 \doteq \mathscr{Y} \backslash \mathcal{T}_{0}$ is connected and

$$
\chi_{\mathcal{T}_{\varepsilon}} \rightarrow \chi_{\mathcal{T}_{0}} \text { in } L^{1}(\mathscr{Y})
$$

and there exists a linear extension operator $Q$ from $H^{1}\left(\mathscr{Y}_{0}\right)$ into $H^{1}(\mathscr{H})$.

Then

i) the sequence $\left\{T_{\varepsilon}\right\}_{\varepsilon}$ is admissible in $\Omega$;

ii) if $A^{\varepsilon}$ is given by (2.4, 2.5), then the whole sequence $\left\{\left(A^{\varepsilon}, T_{\varepsilon}\right)\right\}_{\varepsilon} H^{0}$-converge to some $A^{0}$. The matrix field $A^{0}$ is constant and defined by

$$
A^{0} \lambda=\mathcal{M}_{\mathscr{Y}}\left(\left[A \nabla \widehat{W}_{\lambda}\right]^{\sim}\right), \quad \forall \lambda \in \mathbb{R}^{N},
$$




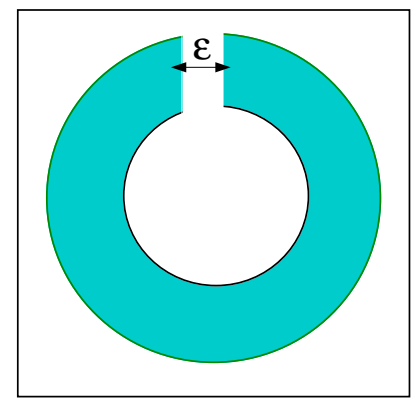

Figure 4

where $\widehat{W}_{\lambda}$ is the unique solution of the problem

$$
\left\{\begin{array}{l}
-\operatorname{div}\left(A(y) \nabla \widehat{W}_{\lambda}\right)=0 \quad \text { in } \mathscr{Y} 0 \\
\left(A(y) \nabla \widehat{W}_{\lambda}\right) \cdot \nu=0 \quad \text { on } \partial \mathcal{T}_{0} \\
\widehat{W}_{\lambda}-\lambda \cdot y \quad \mathscr{Y}-\text { periodic } \\
\mathcal{M}_{\mathscr{Y} 0}\left(\widehat{W}_{\lambda}-\lambda \cdot y\right)=0
\end{array}\right.
$$

and where $\left[A \nabla \widehat{W}_{\lambda}\right] \sim$ denotes the zero extension of $A \nabla \widehat{W}_{\lambda}$ to the whole of $\mathscr{Y}$.

Remark 2.11. Observe that in the case $\mathcal{T}_{\varepsilon} \equiv \mathcal{T}_{0}$, the hypotheses of Theorem 2.10 concerning the holes reduce to the fact that $\mathscr{H}_{0}$ is connected and there exists a linear extension operator $Q$ from $H^{1}(\mathscr{H} /)$ into $H^{1}(\mathscr{Z})$. This is exactly the case of [12]. In the general case, the $A^{0}$ obtained in the theorem is the same as the $H^{0}$-limit of the sequence $\left\{\left(A^{\varepsilon}, T_{\varepsilon}\left(\Omega, \mathcal{T}_{0}\right)\right)\right\}_{\varepsilon}$ corresponding to the case $\mathcal{T}_{\varepsilon} \equiv \mathcal{T}_{0}$.

The solution of (2.11) is understood in the following variational sense:

$$
\left\{\begin{array}{l}
\text { Find } \widehat{W}_{\lambda} \text { such that } \widehat{W}_{\lambda}-\lambda \cdot y \in H \\
\int_{\mathscr{Y} 0} A(y) \nabla \widehat{W}_{\lambda} \nabla \varphi=0 \quad \forall \varphi \in H,
\end{array}\right.
$$

where $H$ is defined by

$$
H \doteq\left\{v \in H_{\mathrm{per}}^{1}\left(\mathscr{Y}_{0}\right), \mathcal{M}_{\mathscr{Y} 0}(v)=0\right\} \cdot
$$

Remark 2.12. Actually, no boundary terms on $\partial \mathscr{Y}$ appear, due to the periodicity and the fact that $\mathscr{Y}$ has the paving property.

As usual, equation (2.12) holds for every $\varphi$ in $H_{\text {per }}^{1}\left(\mathscr{Y}_{0}\right)$.

Remark 2.13. Note that in the hypotheses of Theorem 2.10 the fact that $\mathcal{O} \backslash S$ is connected is necessary and not a consequence of (2.9), not even when $\mathcal{T}_{\varepsilon}$ converges to $\mathcal{T}_{0}$ in the Hausdorff sense (which is stronger than (2.9)) (see Fig. 4).

Question 2.14. It is an open question whether (2.8) together with (2.9) imply the existence of the extension operator $Q$ or at least the connectedness of $\mathscr{Y} 0$.

The proof of Theorem 2.10, including the existence and uniqueness of the solution of (2.11), is given in the following section. 


\section{Poincaré-Wirtinger inequalities and Proof of Theorem 2.10}

We start this section by recalling the following well-known result which concerns the weak convergence of sequences of periodic oscillating functions (see for instance [14], Appendix):

Proposition 3.1. For every $\varepsilon>0$ let $h_{\varepsilon}$ be a $\mathscr{Y}$-periodic function in $L^{p}(\mathscr{H})$ for some $p \in[1,+\infty]$. Consider the sequence $\left\{h_{\varepsilon}\right\}_{\varepsilon}$ defined in $L_{\mathrm{loc}}^{p}\left(\mathbb{R}^{N}\right)$ by $h_{\varepsilon}(x)=h_{\varepsilon}\left(\frac{x}{\varepsilon}\right)$. Then, the following hold:

i) $\left(h_{\varepsilon}\right)$ is bounded in $L_{\mathrm{loc}}^{p}\left(\mathbb{R}^{N}\right)$ if and only if $\left\{h_{\varepsilon}\right\}_{\varepsilon}$ is bounded in $L^{p}(\mathscr{Y})$;

ii) for $1<p<+\infty,\left(h_{\varepsilon}\right)$ converges weakly in $L_{\text {loc }}^{p}\left(\mathbb{R}^{N}\right)$ if and only if the sequence $\mathcal{M}_{\mathscr{F}}\left(h_{\varepsilon}\right)$ is convergent; under this condition, $\left\{h_{\varepsilon}\right\}_{\varepsilon}$ converges weakly to the limit of $\mathcal{M}_{\mathscr{Y}}\left(h_{\varepsilon}\right)$.

Applying this to $h_{\varepsilon}=\chi_{\Omega_{\varepsilon}}$ yields:

Corollary 3.2. Convergence (2.9) of Theorem 2.10, implies that $\chi_{\Omega_{\varepsilon}}$ converges weakly $*$ in $L^{\infty}$ to $\frac{|\mathscr{Y}|}{|\mathscr{Y}|}$, so that (1.4) holds.

The existence of a variational solution of (2.11) requires the Poincaré-Wirtinger inequality for periodic functions, which we recall here:

Definition 3.3. i) The bounded domain $\mathcal{O}$ satisfies the Poincaré-Wirtinger inequality (PWI) if there exists a positive constant $c$ such that

$$
\forall v \in H^{1}(\mathcal{O}), \quad\left\|v-\mathcal{M}_{\mathcal{O}}(v)\right\|_{L^{2}(\mathcal{O})} \leq c\|\nabla v\|_{L^{2}(\mathcal{O})}
$$

The smallest such constant $c$ is denoted $C(\mathcal{O})$.

ii) Suppose that $\mathcal{O}=\mathscr{H} \cap\left(\mathbb{R}^{N} \backslash \mathcal{S}\right)$, where $\mathscr{Y}$ is some connected open set, with piece-wise smooth boundary having the paving property with respect to some basis, and $\mathcal{S}$ is a closed (not necessarily compact) set of $\mathbb{R}^{N}$ included in $\overline{\mathscr{H}}$. We say that $\mathcal{O}$ satisfies the Poincaré-Wirtinger inequality for periodic functions (PPWI) if there exists some constant $c$ such that

$$
\forall v \in H_{\mathrm{per}}^{1}(\mathcal{O}), \quad\left\|v-\mathcal{M}_{\mathcal{O}}(v)\right\|_{L^{2}(\mathcal{O})} \leq c\|\nabla v\|_{L^{2}(\mathcal{O})}
$$

The smallest such constant $c$ is denoted $C_{\text {per }}(\mathcal{O})$.

Remark 3.4. A necessary condition in order to have property (PWI) is that $\mathcal{O}$ be connected. Similarly, a necessary condition in order to have property $(\mathrm{PPWI})$ is that $\Pi(\mathcal{O})$ be connected in the periodic torus associated with the basis $\left(b_{1}, \ldots, b_{N}\right)$.

In the case ii) of Definition 3.3, clearly $C_{\text {per }}(\mathcal{O}) \leq C(\mathcal{O})$.

Proposition 3.5. Let $\mathcal{O}$ be an open set of $\mathbb{R}^{N}$ such that the embedding of $H^{1}(\mathcal{O})$ in $L^{2}(\mathcal{O})$ is compact and $S$ be a compact subset of $\mathcal{O}$ such that $\mathcal{O} \backslash S$ is connected. If there exists a continuous linear extension operator $\mathcal{Q} \in \mathcal{L}\left(H^{1}(\mathcal{O} \backslash S), H^{1}(\mathcal{O})\right)$ then, $\mathcal{O} \backslash S$ satisfies $(P W I)$.

A condition which implies the compact embedding in Proposition 3.5 is the existence of a linear continuous extension operator from $H^{1}(\mathcal{O})$ to $H^{1}\left(\mathbb{R}^{N}\right)$. Examples of domains having this extension property are given in Section 4 . 
Proposition 3.5 is obtained as a particular case of the following one:

Proposition 3.6. Let $\mathcal{O}$ an open set of $\mathbb{R}^{N}$ such that the embedding of $H^{1}(\mathcal{O})$ in $L^{2}(\mathcal{O})$ is compact and $\left\{S_{\varepsilon}\right\}_{\varepsilon}$ be a sequence of compact subset of $\mathcal{O}$ such that, for every $\varepsilon, \mathcal{O} \backslash S_{\varepsilon}$ is connected. Suppose that there exists a sequence of continuous linear extension operator $\mathcal{Q}_{\varepsilon} \in \mathcal{L}\left(H^{1}\left(\mathcal{O} \backslash S_{\varepsilon}\right), H^{1}(\mathcal{O})\right)$ and a positive constant $c_{0}$ (independent of $\varepsilon$ ) with

$$
\forall v \in H^{1}\left(\mathcal{O} \backslash S_{\varepsilon}\right),\left\|\mathcal{Q}_{\varepsilon} v\right\|_{H^{1}(\mathcal{O})} \leq c_{0}\|v\|_{H^{1}\left(\mathcal{O} \backslash S_{\varepsilon}\right)} .
$$

If there exists a compact set $S$ in $\mathcal{O}$ with $\mathcal{O} \backslash S$ connected, and for which

$$
\chi_{S_{\varepsilon}} \rightarrow \chi_{S} \quad \text { in } L^{1}(\mathcal{O})
$$

then, $\mathcal{O} \backslash S_{\varepsilon}$ satisfies the Poincaré-Wirtinger inequality with a constant $C\left(\mathcal{O} \backslash S_{\varepsilon}\right)$ bounded with respect to $\varepsilon$.

Proof. The proof goes by contradiction. Assuming the conclusion does not holds, and using a subsequence which we still denote by $\varepsilon$, there exists a sequence $\left\{u_{\varepsilon}\right\}_{\varepsilon}$ which satisfies

$$
\left\{\begin{array}{cl}
i) & u_{\varepsilon} \in H^{1}\left(\mathcal{O} \backslash S_{\varepsilon}\right), \\
i i) & \left\|u_{\varepsilon}\right\|_{L^{2}\left(\mathcal{O} \backslash S_{\varepsilon}\right)}=1, \\
i i i) & \int_{\mathcal{O} \backslash S_{\varepsilon}} u_{\varepsilon} \mathrm{d} x=0, \\
i v) & \left\|\nabla u_{\varepsilon}\right\|_{L^{2}\left(\mathcal{O} \backslash S_{\varepsilon}\right)} \rightarrow 0 .
\end{array}\right.
$$

By hypothesis, the sequence $\left\{w_{\varepsilon} \doteq \mathcal{Q}_{\varepsilon}\left(u_{\varepsilon}\right)\right\}_{\varepsilon}$ is bounded in $H^{1}(\mathcal{O})$ so that we can assume (up to the extraction of a subsequence) that it converges to some $w$ weakly in $H^{1}(\mathcal{O})$ and, by compact embedding, strongly in $L^{2}(\mathcal{O})$. On the one hand

$$
\int_{\mathcal{O}}\left(1-\chi_{S_{\varepsilon}}\right) w_{\varepsilon}^{2} \mathrm{~d} x=\int_{\mathcal{O}} u_{\varepsilon}^{2} \mathrm{~d} x=1
$$

Observe that (3.1) implies the weak $*$-convergence in $L^{\infty}(\mathcal{O})$. Hence, passing to the limit in (3.3) and using the strong convergence in $L^{2}(\mathcal{O})$ of $\left\{w_{\varepsilon}\right\}_{\varepsilon}$, we conclude that

$$
\int_{\mathcal{O} \backslash S} w^{2} \mathrm{~d} x=1
$$

On the other hand, we now show that $w$ vanishes on $\mathcal{O} \backslash S$ which contradicts (3.4).

First, the equality $0=\int_{\mathcal{O} \backslash S_{\varepsilon}} u_{\varepsilon} \mathrm{d} x=\int_{\mathcal{O}}\left(1-\chi_{S_{\varepsilon}}\right) w_{\varepsilon} \mathrm{d} x$ gives

$$
\int_{\mathcal{O} \backslash S} w \mathrm{~d} x=0
$$

at the limit.

Moreover, from (3.2)iv), for every $\Phi \in(\mathcal{D}(\mathcal{O}))^{N}$

$$
\left|\int_{\mathcal{O}}\left(1-\chi_{S_{\varepsilon}}\right) \Phi \cdot \nabla w_{\varepsilon} \mathrm{d} x\right| \leq c|| \nabla u_{\varepsilon} \|_{L^{2}\left(\mathcal{O} \backslash S_{\varepsilon}\right)} \rightarrow 0
$$

But

$$
0=\lim _{\varepsilon \rightarrow 0} \int_{\mathcal{O}}\left(1-\chi_{S_{\varepsilon}}\right) \Phi \cdot \nabla w_{\varepsilon} \mathrm{d} x=\int_{\mathcal{O}}\left(1-\chi_{S}\right) \Phi \cdot \nabla w \mathrm{~d} x
$$

by $(3.1)$ and since $\nabla w_{\varepsilon}$ converges weakly to $\nabla w$ in $L^{2}(\mathcal{O})$. Consequently, $\nabla w$ vanishes on $\mathcal{O} \backslash S$. By connectedness of $\mathcal{O} \backslash S$ one concludes that $w$ is constant on that set. One completes the proof with (3.5). 
Corollary 3.7. Under the assumptions of Proposition 3.6, for every $\varepsilon$ there exists an extension operator $\mathscr{P}_{\varepsilon}$ from $H^{1}\left(\mathcal{O} \backslash S_{\varepsilon}\right)$ to $H^{1}(\mathcal{O})$ having the following properties for some positive $c_{1}$ :

$$
\begin{cases}i) & \mathscr{P}_{\varepsilon} \in \mathcal{L}\left(H^{1}\left(\mathcal{O} \backslash S_{\varepsilon}\right), H^{1}(\mathcal{O})\right) \\ i i) & \left(\mathscr{P}_{\varepsilon} v\right)_{\left.\right|_{\mathcal{O} \backslash S_{\varepsilon}}}=v \quad \forall v \in H^{1}\left(\mathcal{O} \backslash S_{\varepsilon}\right), \\ i i i) \quad & \left\|\nabla\left(\mathscr{P}_{\varepsilon} v\right)\right\|_{\left(L^{2}(\mathcal{O})\right)^{N}} \leq c_{1}\|\nabla v\|_{\left(L^{2}\left(\mathcal{O} \backslash S_{\varepsilon}\right)\right)^{N}}, \quad \forall v \in H^{1}\left(\mathcal{O} \backslash S_{\varepsilon}\right)\end{cases}
$$

Proof. The proof follows the ideas of the periodic case given in [12]. Define, for every $\varepsilon$

$$
\mathscr{P}_{\varepsilon}(v)=\mathcal{Q}_{\varepsilon}\left(v-\mathcal{M}_{\mathcal{O} \backslash S_{\varepsilon}}(v)\right)+\mathcal{M}_{\mathcal{O} \backslash S_{\varepsilon}}(v), \quad \forall v \in H^{1}\left(\mathcal{O} \backslash S_{\varepsilon}\right)
$$

Properties i) and ii) of (3.6) are straightforward; as for iii) we have

$$
\begin{aligned}
\left\|\nabla \mathscr{P}_{\varepsilon}(v)\right\|_{L^{2}(\mathcal{O})} & =\left\|\nabla \mathcal{Q}_{\varepsilon}\left(v-\mathcal{M}_{\mathcal{O} \backslash S_{\varepsilon}}(v)\right)\right\|_{L^{2}(\mathcal{O})} \\
& \leq\left\|\mathcal{Q}_{\varepsilon}\left(v-\mathcal{M}_{\mathcal{O} \backslash S_{\varepsilon}}(v)\right)\right\|_{H^{1}(\mathcal{O})} \leq c_{0}\left\|v-\mathcal{M}_{\mathcal{O} \backslash S_{\varepsilon}}(v)\right\|_{H^{1}\left(\mathcal{O} \backslash S_{\varepsilon}\right)} \\
& \leq c_{0}\left(1+C\left(\mathcal{O} \backslash S_{\varepsilon}\right)\right)\left\|\nabla\left(v-\mathcal{M}_{\mathcal{O} \backslash S_{\varepsilon}}(v)\right)\right\|_{L^{2}\left(\mathcal{O} \backslash S_{\varepsilon}\right)} \\
& =c_{0}\left(1+C\left(\mathcal{O} \backslash S_{\varepsilon}\right)\right)\|\nabla(v)\|_{L^{2}\left(\mathcal{O} \backslash S_{\varepsilon}\right)} .
\end{aligned}
$$

Let us prove now the existence of the solution of problem (2.11):

Proposition 3.8. Let $\mathscr{Y}$ be a connected open set, with piece-wise smooth boundary, having the paving property with respect to the basis $\left(b_{1}, \ldots, b_{N}\right)$. Suppose that $S$ is a closed (not necessarily compact) set of $\mathbb{R}^{N}$, contained in $\overline{\mathscr{Y}}$ such that $\mathscr{Y} \cap\left(\mathbb{R}^{N} \backslash S\right)$ satisfies the Poincaré-Wirtinger inequality for periodic functions (PPWI). Let $A$ be in $M(\alpha, \beta, \mathscr{Y})$. Then, for every $\lambda \in \mathbb{R}^{N}$ the problem

$$
\left\{\begin{array}{l}
-\operatorname{div}\left(A(y) \nabla \widehat{W}_{\lambda}\right)=0 \quad \text { in } \mathscr{Y} \cap\left(\mathbb{R}^{N} \backslash S\right) \\
\left(A(y) \nabla \widehat{W}_{\lambda}\right) \cdot \nu=0 \quad \text { on } \partial S \\
\widehat{W}_{\lambda}-\lambda \cdot y \quad \mathscr{Y}-\text { periodic } \\
\mathcal{M}_{\mathscr{Y} \cap\left(\mathbb{R}^{N} \backslash S\right)}\left(\widehat{W}_{\lambda}-\lambda \cdot y\right)=0
\end{array}\right.
$$

has a unique solution $\widehat{W}_{\lambda}$ in the following variational sense:

$$
\left\{\begin{array}{l}
\text { Find } \widehat{W}_{\lambda} \text { such that } \widehat{W}_{\lambda}-\lambda \cdot y \in H \\
\int_{\mathscr{Y} \cap\left(\mathbb{R}^{N} \backslash S\right)} A(y) \nabla \widehat{W}_{\lambda} \nabla \varphi=0 \quad \forall \varphi \in H,
\end{array}\right.
$$

where $H$ is the space

$$
H \doteq\left\{v \in H_{\text {per }}^{1}\left(\mathscr{H} \cap\left(\mathbb{R}^{N} \backslash S\right)\right), \mathcal{M}_{\mathscr{Y} \cap\left(\mathbb{R}^{N} \backslash S\right)}(v)=0\right\}
$$

Proof. Set $\widetilde{\eta}_{\lambda}=\lambda \cdot y-\widehat{W}_{\lambda}$ which belongs to the space $H$ defined by (3.9). Hence, (3.7) can be rewritten as

$$
\left\{\begin{array}{l}
-\operatorname{div}\left(A(y) \nabla \widetilde{\eta}_{\lambda}\right)=-\operatorname{div}(A(y) \lambda) \quad \text { in } \mathscr{Y} \cap\left(\mathbb{R}^{N} \backslash S\right), \\
\left(A(y) \nabla \widetilde{\eta}_{\lambda}\right) \cdot \nu=A(y) \lambda \cdot \nu \quad \text { on } \partial S \\
\widetilde{\eta}_{\lambda} \mathscr{Y}-\text { periodic, } \\
\mathcal{M}_{\mathscr{Y} \cap\left(\mathbb{R}^{N} \backslash S\right)}\left(\widetilde{\eta}_{\lambda}\right)=0 .
\end{array}\right.
$$


The variational formulation for $(3.7)$ is then

$$
\left\{\begin{array}{l}
\text { Find } \widetilde{\eta}_{\lambda} \in H \\
\int_{\mathscr{Y} \cap\left(\mathbb{R}^{N} \backslash S\right)} A(y) \nabla \widetilde{\eta}_{\lambda} \nabla \varphi \mathrm{d} y=\int_{\mathscr{Y} \cap\left(\mathbb{R}^{N} \backslash S\right)} A(y) \lambda \nabla \varphi \mathrm{d} y \quad \forall \varphi \in H .
\end{array}\right.
$$

This problem has a unique solution via Lax-Milgram's theorem, because for $\varphi \in H$ we have

$$
\begin{aligned}
\|\varphi\|_{L^{2}\left(\mathscr{Y} \cap\left(\mathbb{R}^{N} \backslash S\right)\right)}^{2} & \leq C_{\text {per }}\left(\mathscr{Y} \cap\left(\mathbb{R}^{N} \backslash S\right)\right)\|\nabla \varphi\|_{L^{2}\left(\mathscr{Y} \cap\left(\mathbb{R}^{N} \backslash S\right)\right)}^{2} \\
& \leq C_{\text {per }}\left(\mathscr{Y} \cap\left(\mathbb{R}^{N} \backslash S\right)\right) \frac{1}{\alpha} \int_{\mathscr{Y} \cap\left(\mathbb{R}^{N} \backslash S\right)} A(y) \nabla \varphi \nabla \varphi \mathrm{d} y .
\end{aligned}
$$

Proof of Theorem 2.10. By Proposition 3.5, Theorem 2.10 is a corollary of Theorem 3.9 below, in which the existence of an extension operator for $\mathscr{H}_{0}$ is replaced by (PPWI).

Theorem 3.9. Under Assumption 2.5, let $\Omega_{\varepsilon}$ be defined by (2.6) and $A^{\varepsilon}$ by (2.4, 2.5). Suppose that for every $\varepsilon$ there exists a linear extension operator $\mathcal{Q}_{\varepsilon}$ from $H^{1}\left(\mathscr{Y}_{\varepsilon}\right)$ to $H^{1}(\mathscr{Y})$ satisfying (2.8), i.e.

$$
\forall \varepsilon, \forall v \in H^{1}(\mathscr{Y} \varepsilon), \quad\left\|\mathcal{Q}_{\varepsilon} v\right\|_{H^{1}(\mathscr{H})} \leq c_{0}\|v\|_{H^{1}(\mathscr{Y} \varepsilon)},
$$

for some positive $c_{0}$. Suppose furthermore that there exists a compact set $\mathcal{T}_{0}$ in $\mathscr{Y}$ such that $\mathscr{Y}_{0} \doteq \mathscr{Y} \backslash \mathcal{T}_{0}$ satisfies the Poincaré-Wirtinger inequality for periodic functions (PPWI) and for which (2.9) holds, i.e.

$$
\chi_{\mathcal{T}_{\varepsilon}} \rightarrow \chi_{\mathcal{T}_{0}} \text { in } L^{1}(\mathscr{Y})
$$

Then the sequence $\left\{T_{\varepsilon}\right\}_{\varepsilon}$ is admissible in $\Omega$ and the whole sequence $\left\{\left(A^{\varepsilon}, T_{\varepsilon}\right)\right\}_{\varepsilon} H^{0}$-converge to the matrix field $A^{0}$ given by (2.10, 2.11).

The proof of Theorem 3.9, which is given at the end of this section, follows the original Tartar's method of oscillating test functions (see [11] for a detailed presentation). In this framework, the test functions $\widehat{W}_{\lambda}^{\varepsilon}$, defined in the reference cell $\mathscr{H}_{\varepsilon}$ depends upon $\varepsilon$. The following proposition gives their precise definition and the properties of the corresponding rescaled functions $\widehat{w}_{\lambda}^{\varepsilon}$ :

Proposition 3.10. Under the assumptions of Theorem 3.9, for every $\lambda \in \mathbb{R}^{N}$, there exists a unique solution $\widehat{W_{\lambda}^{\varepsilon}}$ of

Setting

$$
\left\{\begin{array}{l}
-\operatorname{div}\left(A(y) \nabla \widehat{W}_{\lambda}^{\varepsilon}\right)=0 \quad \text { in } \mathscr{Y} \varepsilon \\
\left(A(y) \nabla \widehat{W}_{\lambda}^{\varepsilon}\right) \cdot \nu=0 \quad \text { on } \partial \mathcal{T}_{\varepsilon} \\
\widehat{W}_{\lambda}^{\varepsilon}-\lambda \cdot y \quad \mathscr{Y}-\text { periodic } \\
\mathcal{M}_{\mathscr{Y} \varepsilon}\left(\widehat{W}_{\lambda}^{\varepsilon}-\lambda \cdot y\right)=0 .
\end{array}\right.
$$

then we have

$$
\widehat{w}_{\lambda}^{\varepsilon}(x)=\varepsilon \widehat{W}_{\lambda}^{\varepsilon}\left(\frac{x}{\varepsilon}\right), \quad \text { on } \Omega_{\varepsilon}
$$

$$
\left\{\begin{array}{l}
{\left[\nabla \widehat{w}_{\lambda}^{\varepsilon}\right]^{\sim} \rightarrow \frac{|\mathscr{Y}|}{|\mathscr{Y}|} \mathcal{M}_{\mathscr{Y} 0}\left(\nabla \widehat{W}_{\lambda}\right) \quad \text { weakly in }\left(L^{2}(\Omega)\right)^{N},} \\
A^{\varepsilon}\left[\nabla \widehat{w}_{\lambda}^{\varepsilon}\right]^{\sim} \rightarrow \frac{|\mathscr{Y}|}{|\mathscr{Y}|} \mathcal{M}_{\mathscr{H}_{0}}\left(A \nabla \widehat{W}_{\lambda}\right) \quad \text { weakly in }\left(L^{2}(\Omega)\right)^{N},
\end{array}\right.
$$

where $\widehat{W}_{\lambda}$ is the unique solution of (2.11).

Proof. The existence of a unique solution of (2.11) is given by Proposition 3.8. By Proposition 3.5, the existence of the unique solution of (3.11) is also given by Proposition 3.8, written for $S=\mathcal{T}_{\varepsilon}$. 
Define $\widetilde{\eta}_{\lambda}^{\varepsilon}=\lambda \cdot y-\widehat{W}_{\lambda}^{\varepsilon}$, which satisfies

$$
\int_{\mathscr{P} / \varepsilon} A(y) \nabla \widetilde{\eta}_{\lambda}^{\varepsilon} \nabla \varphi=\int_{\mathscr{P} / \varepsilon} A(y) \lambda \nabla \varphi \quad \forall \varphi \in H_{\varepsilon}
$$

where $H_{\varepsilon}$ is defined by

$$
H_{\varepsilon} \doteq\left\{v \in H_{\mathrm{per}}^{1}\left(\mathscr{Y}_{\varepsilon}\right), \mathcal{M}_{\mathscr{Y} \varepsilon}(v)=0\right\}
$$

With the choice $\varphi=\widetilde{\eta}_{\lambda}^{\varepsilon}$ in (3.14) and by (2.5), one concludes that

$$
\left\|\nabla \widetilde{\eta}_{\lambda}^{\varepsilon}\right\|_{L^{2}(\mathscr{Y} \varepsilon)} \leq C .
$$

Let $\mathscr{P}_{\varepsilon}$ be the extension operator from $H^{1}(\mathscr{Y} \varepsilon)$ to $H^{1}(\mathscr{Y})$ given by Corollary 3.7. From (3.16) and (3.6), up to a subsequence, we can assume that there exists a subsequence (still denoted $\varepsilon$ ) and a function $\omega_{\lambda} \in H^{1}(\mathscr{Y})$ such that

We claim that:

$$
\mathscr{P}_{\varepsilon} \widehat{W}_{\lambda}^{\varepsilon} \rightarrow \omega_{\lambda} \text { weakly in } H^{1}(\mathscr{Y}) \text {. }
$$

$$
\omega_{\lambda \mid}=\widehat{W}_{\lambda} \text {. }
$$

The $\mathscr{Y}$-periodicity of $\omega_{\lambda}-\lambda \cdot y$ follows, together with (3.17), from that of $\left(\mathscr{P}_{\varepsilon} \widehat{W}_{\lambda}^{\varepsilon}-\lambda \cdot y\right)$, itself a consequence of the $\mathscr{Y}_{\varepsilon}$-periodicity of $\widehat{W}_{\lambda}^{\varepsilon}-\lambda \cdot y$ and the compactness of $\mathcal{T}_{\varepsilon}$ in $\mathscr{Y}$.

The fact that $\mathcal{M}_{\mathscr{Y} 0}\left(\omega_{\lambda}-\lambda \cdot y\right)=0$ follows from

$$
\int_{\mathscr{H} 0} \omega_{\lambda}-\lambda \cdot y=\int_{\mathscr{H}} \chi_{\mathscr{Y} /}\left(\omega_{\lambda}-\lambda \cdot y\right)=\lim _{\varepsilon \rightarrow 0} \int_{\mathscr{Y}} \chi_{\mathscr{Y} \varepsilon}\left(\mathscr{P}_{\varepsilon} \widehat{W}_{\lambda}^{\varepsilon}-\lambda \cdot y\right)=0,
$$

where we used (2.9) and (3.17).

Finally, let $\varphi$ be a smooth $\mathscr{Z}$-periodic function. According to Remark 2.12, we have

$$
0=\int_{\mathscr{Q} / \varepsilon} A(y) \nabla \widehat{W}_{\lambda}^{\varepsilon}(y) \nabla \varphi(y)=\int_{\mathscr{Y}} \chi_{\mathscr{H} \varepsilon} A(y) \nabla\left(\mathscr{P} \widehat{W_{\lambda}^{\varepsilon}}\right)(y) \nabla \varphi(y) .
$$

Passing to the limit as $\varepsilon \rightarrow 0$ while using (3.17) and Assumptions (2.9), yields

$$
0=\int_{\mathscr{Y} / 0} \chi_{\mathscr{Z}} A(y) \nabla \omega_{\lambda}(y) \nabla \varphi(y) \mathrm{d} y=\int_{\mathscr{Y} 0} A(y) \nabla \omega_{\lambda}(y) \nabla \varphi(y) \mathrm{d} y .
$$

This means that $\omega_{\lambda \mid}$ is the solution of (2.11), so that, by uniqueness and by weak compactness, we get (3.18).

Set now $h_{\varepsilon}=\chi_{\mathscr{Y} \varepsilon} \nabla \mathscr{P}_{\varepsilon} \widehat{W}_{\lambda}^{\varepsilon}$. Using convergences (2.9) and (3.17) together with (3.18), we get

$$
\begin{aligned}
& \mathcal{M}_{\mathscr{H}}\left(h_{\varepsilon}\right)=\frac{1}{|\mathscr{Y}|} \int_{\mathscr{Y}} \chi_{\mathscr{Y} \varepsilon} \nabla \mathscr{P}_{\varepsilon} \widehat{W}_{\lambda}^{\varepsilon}(y) \mathrm{d} y \rightarrow \frac{1}{|\mathscr{Y}|} \int_{\mathscr{Y}} \chi_{\mathscr{H} /} \nabla \omega_{\lambda}(y) \mathrm{d} y \\
& =\frac{1}{|\mathscr{Y}|} \int_{\mathscr{Y} 0} \nabla \widehat{W}_{\lambda}(y) \mathrm{d} y=\frac{|\mathscr{H}|}{|\mathscr{Y}|} \mathcal{M}_{\mathscr{H} 0}\left(\nabla \widehat{W}_{\lambda}\right) .
\end{aligned}
$$

Then, Proposition 3.1, for this $h_{\varepsilon}$ yields the first convergence in (3.13). The second convergence of (3.13) follow similarly from the choice $\ell_{\varepsilon}=\chi_{\mathscr{Y} \varepsilon} A \nabla \mathscr{P}_{\varepsilon} \widehat{W}_{\lambda}^{\varepsilon}$.

Proof of Theorem 3.9. Admissibility follows from Corollary 3.7 for $\mathcal{O}=\mathcal{Y}$ and $S_{\varepsilon}=\mathcal{T}_{\varepsilon}$, together with Corollary 3.2 and Corollary 2.9i). 
Let $f$ be given in $L^{2}(\Omega)$, and $u_{\varepsilon}$ the solution of

$$
\left\{\begin{array}{l}
-\operatorname{div}\left(A^{\varepsilon} \nabla u_{\varepsilon}\right)=f \quad \text { in } \Omega_{\varepsilon} \\
\left(A^{\varepsilon} \nabla u_{\varepsilon}\right) \cdot \nu=0 \\
u_{\varepsilon}=0 \quad \text { on } \partial \Omega .
\end{array}\right.
$$

By Remark 2.3 and Theorem 2.4, there exists a subsequence (still denoted $\{\varepsilon\}$ ) and a matrix $A^{0}$ such that

$$
P_{\varepsilon} u_{\varepsilon} \rightarrow u \quad \text { weakly in } H_{0}^{1}(\Omega),
$$

where $u$ is the unique solution of

$$
\left\{\begin{array}{l}
-\operatorname{div}\left(A^{0} \nabla u\right)=\theta f \quad \text { in } \Omega \\
u=0 \quad \text { on } \partial \Omega
\end{array}\right.
$$

and $\theta=\frac{|\mathscr{Y}|}{|\mathscr{Y}|}$, due to Corollary 3.2.

At this point, to obtain the claimed formula for $A^{0}$, it suffices to use Proposition 3.10 in the method of oscillating test functions, with $\varphi \widehat{w}_{\lambda}^{\varepsilon}$ as test function in problem (1.1), where $\varphi$ is in $\mathcal{D}(\Omega)$ and

$$
\widehat{w}_{\lambda}^{\varepsilon}(x)=\varepsilon \widehat{W}_{\lambda}^{\varepsilon}\left(\frac{x}{\varepsilon}\right) \quad \text { on } \Omega_{\varepsilon}
$$

\section{Questions 3.11.}

- Are there reasonable conditions under which

$$
\left.\mathscr{P}_{\varepsilon} \widehat{W}_{\lambda}^{\varepsilon}\right|_{\mathscr{Y} 0} \rightarrow \widehat{W}_{\lambda} \quad \text { strongly in } H^{1}\left(\mathscr{Y}_{0}\right) ?
$$

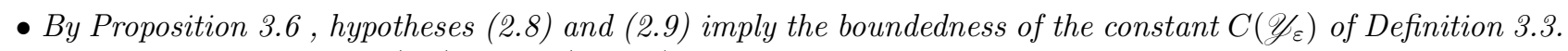
Does the boundedness of $C\left(\mathscr{H}_{\varepsilon}\right)$ imply (PPWI) for $\mathscr{H}_{0}$ ?

\section{Domains For Which the EXTEnsion PROPERTy HOLDS}

One of the main assumptions in Theorem 2.10 (and in the related results) is the existence of an extension operator. The purpose of this paragraph is to present some sufficient conditions for a domain $\mathcal{O}$ of $R^{N}$ to have this property. Classically, this property is used to establish important results concerning Sobolev spaces, such as the density of smooth functions and Sobolev embeddings (including compactness).

Definition 4.1. For $p \in[1, \infty]$, the domain $\mathcal{O}$ has the $p$-extension property whenever there is a bounded linear extension operator from $W^{1, p}(\mathcal{O})$ to $W^{1, p}\left(R^{N}\right)$. have:

This property is known to be connected to the regularity of the boundary of the domain. More precisely, we

Theorem 4.2 (Calderon-Stein, see Stein [29]). If $\partial \mathcal{O}$ has the locally uniform cone property, then it has the p-uniform extension property for every $p$.

It is known that the locally uniform cone-property is equivalent to having a Lipschitz boundary (see Chenais [10]).

There are simple examples (in dimension 2) of domains which have cusps and do not have the $p$-extension property (see Maz'ja [23]). On the other hand, one can wonder if some fractal behaviors of the boundary are compatible with the $p$-extension property.

As far as we know, in this direction the following definition, due to Jones, of $(\varepsilon, \delta)$-domains (now called Jones-domains), gives the most general sufficient condition for the extension property. These domains were also introduced independently in Martio [20] as uniform domains with a somewhat different definition. 
Definition 4.3 (see Jones [19]). For given positive $\varepsilon$ and $\delta, \mathcal{O}$ is an $(\varepsilon, \delta)$ Jones-domain whenever for every $x$ and $y$ in $\mathcal{O}$ with $d(x, y)<\delta$, there is a rectifiable arc $\gamma$ in $\mathcal{O}$ satisfying:

$$
\ell(\gamma) \leq \frac{1}{\varepsilon} d(x, y), \text { and } d(z, \partial \mathcal{O}) \geq \frac{\varepsilon d(x, z) d(y, z)}{d(x, y)} \text { for all } z \in \gamma,
$$

where $d$ denotes the Euclidian distance in $R^{N}$ and $\ell(\gamma)$ the length of the arc.

The notion of Jones-domain is related to the geometry of the boundary of the domain, and in some sense prevents the presence of too many or intricate spikes.

The following result is then proved:

Theorem 4.4 [19]. Let $\mathcal{O}$ be an $(\varepsilon, \delta)$ Jones-domain in $R^{N}$. Then $\mathcal{O}$ has the uniform p-extension property for every $p$. Moreover, the norm of the extension operator is bounded above by a number which only depends upon $\varepsilon, \delta$ and $N$.

A similar result holds for extensions on the spaces $W^{k, p}(\mathcal{O}), k>1$.

In the case of dimension 2, things are somewhat simpler. First, for bounded domains (which we are considering here), $(\varepsilon, \delta)$ Jones-domains are the same as $(\varepsilon, \infty)$ Jones-domains. Then, a simply connected domain $\mathcal{O}$, locally on one side of its boundary (in other words, the boundary of the domain is a Jordan curve), is an $(\varepsilon, \infty)$ Jonesdomain if and only if the complement of its closure is also an $(\varepsilon, \infty)$ Jones-domain. It turns out also that the $p$-condition of Theorem 4.4 is essentially necessary:

Theorem 4.5 [19]. Let $\mathcal{O}$ be finitely connected in $R^{2}$. Then, $\mathcal{O}$ has the p-uniform extension property for every $p$ if and only if it is an $(\varepsilon, \delta)$ Jones-domain, for some positive $\varepsilon$ and $\delta$.

Here, $\mathcal{O}$ finitely connected means that its complement in $R^{N}$ has finitely many connected components.

Actually, an interesting example of $(\varepsilon, \infty)$-domain is given in the plane by the well-known snowflake domain of Koch (see Fig. 5), as well as its complement (in a larger ball). These two domains are clearly not with Lipschitz boundary, but they still have the $p$-extension property for every $p$.

Also, any element of the usual sequence approaching one of these domains is an $(\varepsilon, \infty)$-domain. Hence,

Corollary 4.6. Theorem 2.10 applies for the sequence $\left\{\mathcal{T}_{\varepsilon}\right\}$ approaching the plane snowflake domain of Koch (as well as for the snowflake itself!).

However, Theorem 4.5 is not true for higher dimensions, since in dimension 3 there are domains with the $p$-uniform extension property which are not $(\varepsilon, \delta)$ Jones-domains for any values of $\varepsilon$ and $\delta$ (see [19]).

This leaves open the question of $p$-extension properties for such domains derived in $\mathbb{R}^{3}$ in similar way as the Koch snowflake (the three-dimensional snowflakes).

It is a conjecture that the bounded component $\mathcal{O}$ of the (hyper)-snowflake in $\mathbb{R}^{N}$ is a Jones-domain for some $(\varepsilon, \delta)$, but not its complement, which, in our framework, is the interesting domain, $\mathcal{O}$ being the hole.

\section{The CASe Without EXTEnsion PROPERTy}

In this section, we go beyond of the framework of the $H_{0}$-convergence by not assuming the existence of the extension operators as in Theorem 2.10.

We have in mind the following two cases. The first one is when Assumption 2.5 holds but there exists no family satisfying (2.8). This can be due to a lack of regularity of the boundary of the $\mathcal{T}_{\varepsilon}$ (no extension operators), or to its increasing complexity (no uniform bound for existing extension operators).

The second one concerns the case where $\mathcal{T}_{\varepsilon}$ is not compact in $\mathscr{H}$. For example, one can consider fibers in $\mathbb{R}^{3}$ or some reticulated structures (see for instance Bakhvalov-Panasenko [4], Briane [7], Cioranescu-Saint Jean Paulin [13]).

In this situation, contrary to the case of Section 3, where the existence of (uniform) extension operators together with the Poincaré inequality in $H_{0}^{1}(\Omega)$ insures the existence and uniform estimates for the solution $u_{\varepsilon}$, 

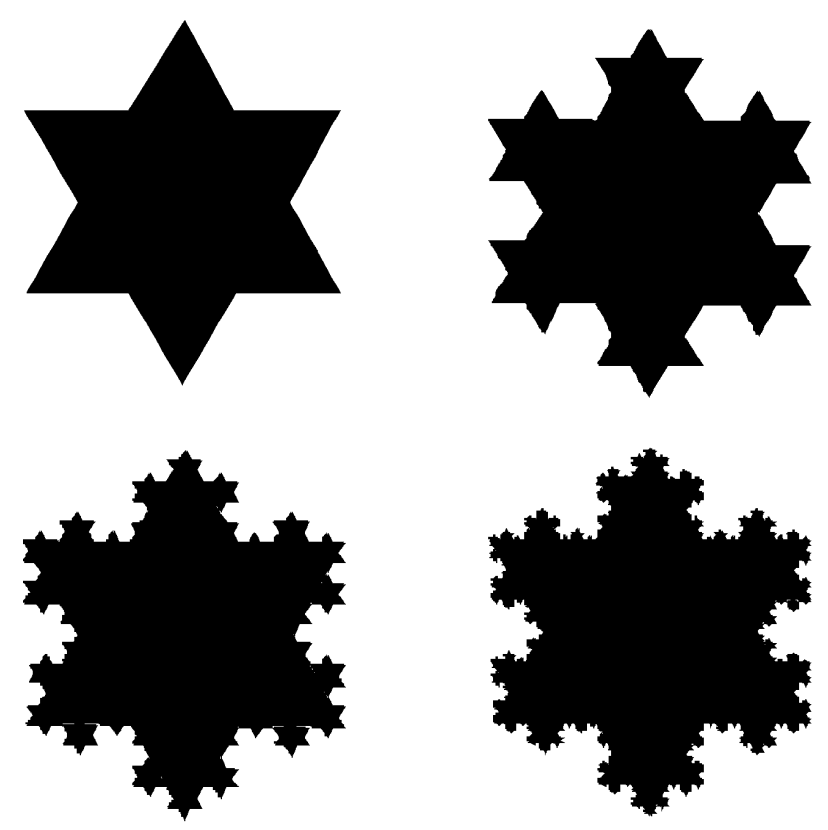

FiguRE 5

neither the existence nor uniform estimates for the $H_{0}^{1}\left(\Omega_{\varepsilon}\right)$ norm of solutions are straightforward. The simplest way to avoid this difficulty, is to add a zero order term in the equation of the form $a u_{\varepsilon}$ with a strictly positive constant $a$. Once this is take care of, the next (and more interesting) question is how to pass to the limit and justify formulas.

Even though they are connected, these two questions are different in nature. Indeed, as we will see below, the first one relies on Poincaré type inequality whereas the second one makes essentially use of the PoincaréWirtinger inequality for periodic functions. We propose below to deal with each question separately.

We introduce the following geometrical hypothesis, which is more general than Assumption 2.5 (we still use notations $(2.1-2.3))$.

Assumption 5.1. A basis $\left(b_{1}, \ldots, b_{N}\right)$ is given in $\mathbb{R}^{N}$. Let $\left\{\mathcal{T}_{\varepsilon}\right\}_{\varepsilon}$ be a sequence of compact sets of $\mathbb{R}^{N}$, such that either for every $\varepsilon, \mathcal{T}_{\varepsilon}$ is the closure of its interior, or for every $\varepsilon, \mathcal{T}_{\varepsilon}$ has zero Lebesgue measure. We assume that there exists a connected open set $\mathscr{Y}$, with piece-wise smooth boundary, having the paving property with respect to the basis $\left(b_{1}, \ldots, b_{N}\right)$ and such that

$$
\text { for every } \varepsilon>0, \mathcal{T}_{\varepsilon} \subset \overline{\mathscr{H}} \quad \text { and } \quad \mathscr{Y}_{\varepsilon} \doteq \mathscr{Y} \cap\left(\mathbb{R}^{N} \backslash T_{\varepsilon}\left(\mathbb{R}^{N}, \mathcal{T}_{\varepsilon}\right)\right) \text { is connected. }
$$

Remark 5.2. The assumption that $\mathcal{T}_{\varepsilon}$ has zero Lebesgue measure implies that $\left.\operatorname{Int}\left(\mathcal{T}_{\varepsilon}\right)=\emptyset\right)$ and corresponds to the case of cracks (see Attouch-Murat for an example of periodic homogenization of cracks).

It is easy to check that Assumption 2.5 implies Assumption 5.1.

The following definition extends the notion of $H_{\text {per }}^{1}(\mathscr{Y} \backslash \mathcal{S})$ used till now to the case where $\mathcal{S}$ is not compact in $\mathscr{H}$.

Definition 5.3. Suppose that $\mathcal{O}=\mathscr{H} \cap\left(\mathbb{R}^{N} \backslash \mathcal{S}\right)$, where $\mathscr{H}$ is some connected open set, with piece-wise smooth boundary having the paving property with respect to some basis, and $\mathcal{S}$ is some compact set of $\mathbb{R}^{N}$ included in $\overline{\mathscr{Y}}$. We denote by $H_{\text {per }}^{1}(\mathcal{O})$ the space of $\mathscr{Y}$-periodic functions in $H_{\text {loc }}^{1}\left(\mathbb{R}^{N} \backslash T_{1}\left(\mathbb{R}^{N}, \mathcal{S}\right)\right)$. 
Before stating the main theorem of this section, we introduce some notation and supplementary assumptions.

Assumption 5.4. Let $\mathscr{Y}$ and $\mathcal{T}_{\varepsilon}$ be as in Assumption 5.1. There exists a compact set $\mathcal{T}_{0} \subset \overline{\mathscr{Y}}$ such that every connected component $\mathscr{Y}_{0}^{i}, i \in \mathcal{I}$ of $\mathscr{H}_{0} \doteq \mathscr{Y} \cap\left(\mathbb{R}^{N} \backslash \mathcal{T}_{0}\right)$, satisfies the Poincaré-Wirtinger inequality for periodic functions (PPWI) and

$$
\begin{aligned}
& \text { i) } \quad \chi_{\mathcal{T}_{\varepsilon}} \rightarrow \chi_{\mathcal{T}_{0}} \text { in } L^{1}(\mathscr{Y}) ; \\
& \text { ii) } \quad \text { the Hausdorff excess } e\left(\mathcal{T}_{\varepsilon}, \mathcal{T}_{0}\right) \doteq \sup _{x \in \mathcal{T}_{\varepsilon}} d\left(x, \mathcal{T}_{0}\right) \text { tends to zero, as } \varepsilon \rightarrow 0 \text {. }
\end{aligned}
$$

If for every $\varepsilon, \mathcal{T}_{\varepsilon}$ is the closure of its interior, we also assume that $\mathcal{T}_{0} \subset \overline{\mathscr{Y}}$ is the closure of its interior.

Remark 5.5. The Hausdorff convergence of $\mathcal{T}_{\varepsilon}$ to $\mathcal{T}_{0}$ implies i) and ii) of Assumption 5.4. If for every $\varepsilon$ the set $\mathcal{T}_{\varepsilon}$ has zero Lebesgue measure (case of cracks), then convergence i) implies that $\mathcal{T}_{0}$ has zero Lebesgue measure also.

Assumption 5.6. Let $\mathscr{Y}$ and $\mathcal{T}_{\varepsilon}$ be as in Assumption 5.1. Let $\mathcal{T}_{0} \subset \overline{\mathscr{H}}$ be a compact set which is the closure of its interior and set $\mathscr{Y} 0 \doteq \mathscr{Y} \cap\left(\mathbb{R}^{N} \backslash \mathcal{T}_{0}\right)$. that

For any smooth $\mathscr{Y}_{0}$-periodic function $\varphi$ and for every $\varepsilon$ positive, there exists a function $\varphi_{\varepsilon} \in H_{\text {per }}^{1}\left(\mathscr{Y}_{\varepsilon}\right)$ such

i) $\widetilde{\varphi_{\varepsilon} \mathscr{Y}_{0}}$ converges strongly to $\varphi$ in $L^{2}\left(\mathscr{H}_{0}\right)$;

ii) $\left[\nabla \varphi_{\varepsilon}\right]_{1}^{\sim} \mathscr{Y}_{0}$ converges strongly to $\nabla \varphi$ in $L^{2}\left(\mathscr{H}_{0}\right)$.

Assumption 5.6 is a somewhat natural generalization of a variational convergence of spaces, adapted to the $H_{\text {per }}^{1}\left(\mathscr{Y}_{\varepsilon}\right)$ 's. Some examples where it is satisfied are given in the proposition below.

Proposition 5.7. Suppose that Assumptions 5.1 and 5.4i) hold. Then, Assumption 5.6 is satisfied in each of the following cases:

a) for every $\varepsilon, \mathscr{Y}_{\varepsilon} \subset \mathscr{H}_{0}$;

b) there exists a linear continuous extension operator $\mathscr{P}_{0}$ from $H^{1}\left(\mathscr{H}_{0}\right)$ to $H^{1}(\mathscr{H})$;

c) $\mathscr{Y}_{\varepsilon}$ is obtained from $\mathscr{H}_{0}$ by a smooth deformation $\Psi_{\varepsilon}$ (continuous with respect to $\varepsilon$ ).

Proof. From the classical Lebesgue measure theory, the following choices of $\varphi_{\varepsilon}$ satisfy 4.6 i) and ii) for each case:

a) $\varphi_{\varepsilon}=\varphi_{\mid \mathscr{Y} \varepsilon}$

b) $\varphi_{\varepsilon}=\left(\mathscr{P}_{0} \varphi\right)_{\mid \mathscr{Y} \varepsilon}$

c) $\varphi_{\varepsilon}(x)=\varphi\left(\Psi_{\varepsilon}^{-1}(x)\right)$.

For $f$ given in $L^{2}(\Omega)$, consider the problem

$$
\left\{\begin{array}{l}
-\operatorname{div}\left(A^{\varepsilon} \nabla u_{\varepsilon}\right)+a_{0}^{\varepsilon} u_{\varepsilon}=f \quad \text { in } \Omega_{\varepsilon} \\
\left(A^{\varepsilon}(x) \nabla u_{\varepsilon}\right) \cdot \nu=0 \text { on } \partial T_{\varepsilon} \\
u_{\varepsilon}=0 \text { on } \partial \Omega
\end{array}\right.
$$

where $\Omega_{\varepsilon}$ is defined by $(2.6), A^{\varepsilon}$ by $(2.4,2.5)$ and $\left\{a_{0}^{\varepsilon}\right\}_{\varepsilon}$ is a sequence such that

$$
\left\{\begin{array}{l}
a_{0} \text { is a non-negative } \mathscr{Y}-\text { periodic function in } L^{\infty}(\mathscr{Y}), \\
a_{0}^{\varepsilon}(x)=a_{0}\left(\frac{x}{\varepsilon}\right) \quad \text { a.e. in } \mathbb{R}^{N} .
\end{array}\right.
$$


The following result is straightforward:

Proposition 5.8. For $f$ given in $L^{2}(\Omega)$, let $\Omega_{\varepsilon}$ be defined by (2.6), $A^{\varepsilon}$ by (2.4, 2.5) and $\left\{a_{0}^{\varepsilon}\right\}_{\varepsilon}$ by (5.2), under Assumption 5.1. If $\alpha_{0} \doteq \inf a_{0}>0$, problem (4.1) has a unique solution in the space $\mathcal{V}_{\varepsilon}$ (see (1.2)). Furthermore, there exists a constant c such that

$$
\int_{\Omega_{\varepsilon}}\left(\left|\nabla u_{\varepsilon}\right|^{2}+u_{\varepsilon}^{2}\right) \leq c
$$

Remark 5.9. If the Poincaré inequality holds in the spaces $\mathcal{V}_{\varepsilon}$ with a constant independent of $\varepsilon$, then (5.3) holds, but it is an open problem to characterize this situation with reasonable geometric conditions on the holes, apart from the case where there exists a family of extension operator verifying (1.6). In particular, it would be interesting to clarify the connection between the uniform Poincaré inequality for the spaces $\mathcal{V}_{\varepsilon}$ and the Poincaré-Wirtinger inequality in $\mathscr{Y}_{\varepsilon}$.

Regarding convergence, the following result holds:

Theorem 5.10. Under Assumptions 5.1, 5.4 and 5.6, let $\Omega_{\varepsilon}$ be defined by (2.6) and $A^{\varepsilon}$ by (2.4, 2.5). For $f$ given in $L^{2}(\Omega)$, suppose that for every $\varepsilon, u_{\varepsilon}$ is a solution of (5.1) satisfying (5.3). Suppose further that for every $\varepsilon$, the domain $\mathscr{Y} \varepsilon$ satisfies the Poincaré-Wirtinger inequality and that

$$
\lim _{\varepsilon \rightarrow 0} \varepsilon C(\mathscr{H} \varepsilon)=0
$$

Let $A^{0}$ be the constant matrix field defined by

$$
A^{0} \lambda=\mathcal{M}_{\mathscr{Y}}\left(\left[A \nabla \widehat{W}_{\lambda}\right]^{\sim}\right), \quad \forall \lambda \in \mathbb{R}^{N},
$$

where $\widehat{W}_{\lambda}$ is the unique solution of the problem

$$
\left\{\begin{array}{l}
-\operatorname{div}\left(A(y) \nabla \widehat{W}_{\lambda}\right)=0 \quad \text { in } \mathscr{Y} 0 \\
\left(A(y) \nabla \widehat{W}_{\lambda}\right) \cdot \nu=0 \quad \text { on } \partial \mathcal{T}_{0}, \\
\widehat{W}_{\lambda}-\lambda \cdot y \quad \mathscr{Y}-\text { periodic } \\
\mathcal{M}_{\mathscr{Y}_{0}^{i}}\left(\widehat{W}_{\lambda}-\lambda \cdot y\right)=0, \quad i \in \mathcal{I},
\end{array}\right.
$$

where the $\mathscr{Y}_{0}^{i}, i \in \mathcal{I}$, are the connected components of $\mathscr{H}_{0}$ (recall that $[\cdot]^{\sim}$ denotes the extension by zero to the whole of $\mathscr{\mathscr { H }})$.

Then, $\left\{\widetilde{u_{\varepsilon}}\right\}_{\varepsilon}$ is bounded in $L^{2}(\Omega)$ and every converging subsequence $\left\{\widetilde{u_{\varepsilon^{\prime}}}\right\}_{\varepsilon}$ satisfies

$$
\widetilde{u_{\varepsilon^{\prime}}} \rightarrow \theta u \quad \text { weakly in } L^{2}(\Omega)
$$

where $\theta=\frac{\left|\mathscr{Y} \backslash \mathcal{T}_{0}\right|}{|\mathscr{Y}|}$ and $u$ satisfies

$$
\left\{\begin{array}{l}
u \in H^{1}(\Omega), \\
-\operatorname{div}\left(A^{0} \nabla u\right)+\theta \mathcal{M}_{\mathscr{H}_{0}}\left(a_{0}\right) u=\theta f \quad \text { in } \mathcal{D}^{\prime}(\Omega) .
\end{array}\right.
$$

Furthermore, in the case where $\mathcal{T}_{\varepsilon}$ has zero Lebesgue measure (cracks), $\theta=1$ and the convergence in (5.7) is strong, i.e.

$$
u_{\varepsilon^{\prime}} \rightarrow u \quad \text { strongly in } L^{2}(\Omega)
$$


In Theorem 5.10, the solution of (5.6) is understood in the following variational sense:

$$
\left\{\begin{array}{l}
\text { Find } \widehat{W}_{\lambda} \text { such that } \widehat{W}_{\lambda}-\lambda \cdot y \in H \\
\int_{\mathscr{H} 0} A(y) \nabla \widehat{W}_{\lambda} \nabla \varphi=0 \quad \forall \varphi \in H,
\end{array}\right.
$$

where $H$ is the Hilbert space defined by

$$
\left.H \doteq\left\{v \in L_{\mathrm{loc}}^{2}\left(\mathscr{Y}_{0}\right)\right), \nabla v \in L^{2}\left(\mathscr{H}_{0}\right) \text { and } \forall i \in \mathcal{I}, v \in H_{\mathrm{per}}^{1}\left(\mathscr{Y}_{0}^{i}\right), \mathcal{M}_{\mathscr{Y}_{0}^{i}}(v)=0\right\}
$$

endowed with the norm

$$
\|v\|_{H}=\|\nabla v\|_{L^{2}(\mathscr{H})} .
$$

Remark 5.11. In general, equation (5.8) does not have a unique solution, since there is no boundary condition for $u$. However, among all possible limit points $u$ in $(5.7)$, there is at most one in $H_{0}^{1}(\Omega)$, which is then the unique solution of

$$
\left\{\begin{array}{l}
-\operatorname{div}\left(A^{0} \nabla u\right)+\theta \mathcal{M}_{\mathscr{Y}_{0}}\left(a_{0}\right) u=\theta f \quad \text { in } \Omega \\
u=0 \quad \text { on } \partial \Omega .
\end{array}\right.
$$

A first step in addressing the question in Remark 5.11 is given in Proposition 5.13 below for which we introduce the following assumption:

Assumption 5.12. Under Assumption 5.1, denote

$$
\forall j=1, \cdots, N, \quad \partial \mathscr{Y}_{j}^{ \pm} \doteq \overline{\mathscr{H}} \cap \tau_{ \pm b_{j}}(\mathscr{H}) .
$$

For every $\varepsilon$, there exists a positive constant $C_{\varepsilon}^{\prime}$ such that

$$
\forall u \in H^{1}\left(\mathscr{Y}_{\varepsilon}\right), \forall j=1, \cdots, N, \quad\left\|u-\mathcal{M}_{\mathscr{Y}_{j}^{ \pm}}(u)\right\|_{L^{2}(\mathscr{\mathscr { V }} \varepsilon} \leq C_{\varepsilon}^{\prime}\|\nabla u\|_{L^{2}(\mathscr{Y} \varepsilon)} .
$$

Proposition 5.13. Suppose that Assumption 5.12 holds with $C_{\varepsilon}^{\prime}$ bounded above by some positive constant $C^{\prime}$ and let $\Omega_{\varepsilon}$ be defined by (2.6). Suppose furthermore that the boundary $\partial \Omega$ of $\Omega$ is Lipschitz continuous. Let $\left\{v_{\varepsilon}\right\}_{\varepsilon}$ be a sequence such that, for each $\varepsilon, v_{\varepsilon} \in V_{\varepsilon}$ and $\left\|v_{\varepsilon}\right\|_{V_{\varepsilon}}$ is bounded. Then, every weak limit point in $L^{2}(\Omega)$ of $\left\{\widetilde{v}_{\varepsilon}\right\}_{\varepsilon}$ is actually in $H_{0}^{1}(\Omega)$.

Remark 5.14. For a fixed reference hole contained in $\mathscr{H}$ and having a Lipschitz boundary, a similar result, concerning the limits of the sequence of local averages of $\left\{v_{\varepsilon}\right\}_{\varepsilon}$ is proved in [2] (Lem. 4.3). In this case, Assumption 5.12 readily holds. As far as we know, a theory describing classes of sets satisfying that assumption in the spirit of John domains remains to be studied.

The proof of Proposition 5.13, in the spirit of that of Lemma 4.3 in [2], is given in the Appendix.

Remark 5.15. The elements of $H$ are not necessarily in $L^{2}(\mathscr{H})$ since the constants $C_{\text {per }}\left(\mathscr{Y}_{0}^{i}\right)$ are not bounded in general. If these are bounded, for instance if $\mathscr{Y} 0$ has a finitely many connected components (i.e. the set $\mathcal{I}$ is finite), then the space $H$ of (5.11) is simply

$$
\left\{v \in H_{\mathrm{per}}^{1}\left(\mathscr{Y}_{0}\right), \quad \forall i \in \mathcal{I}, \mathcal{M}_{\mathscr{Y}_{0}^{i}}(v)=0\right\}
$$

The proof of Theorem 5.10, as well as the existence and uniqueness of the solution of (5.6), are given in Section 6 .

Some examples where Theorem 5.10 applies can be found in [7], where it is shown that condition (5.4) is optimal. A class of domains for which the results of this section can be applied is given in Section 7 (Johndomains). 


\section{Proofs of the Results of Section 5}

The following proposition generalizes Proposition 3.8 to the case where $\mathscr{H}_{0}$ is not connected:

Proposition 6.1. Let $\mathscr{Y}$ be a connected open set with piece-wise smooth boundary, having the paving property with respect to some basis, and $A$ in $M(\alpha, \beta, \mathscr{Y})$. Let $\mathcal{S}$ be a compact set of $\mathbb{R}^{N}$ included in $\overline{\mathscr{Y}}$, which is the closure of its interior and such that such that every connected component $\mathscr{Y}_{s}^{i}$, $i \in \mathcal{I}$, of $\mathscr{Y} \cap\left(\mathbb{R}^{N} \backslash S\right)$ satisfies the Poincaré-Wirtinger inequality for periodic functions (PPWI).

Then, for every $\lambda \in \mathbb{R}^{N}$ the problem

$$
\left\{\begin{array}{l}
-\operatorname{div}\left(A(y) \nabla \widehat{W}_{\lambda}\right)=0 \quad \text { in } \mathscr{Y} \cap\left(\mathbb{R}^{N} \backslash S\right), \\
\left(A(y) \nabla \widehat{W}_{\lambda}\right) \cdot \nu=0 \quad \text { on } \mathscr{Y} \cap \partial S \\
\widehat{W}_{\lambda}-\lambda \cdot y \quad \mathscr{Y}-\text { periodic } \\
\mathcal{M}_{\mathscr{Y}_{s}^{i}}\left(\widehat{W}_{\lambda}-\lambda \cdot y\right)=0, \quad \forall i \in \mathcal{I}
\end{array}\right.
$$

has a unique solution $\widehat{W}_{\lambda} \in \hat{H}$ in the following variational sense:

$$
\left\{\begin{array}{l}
\text { Find } \widehat{W}_{\lambda} \text { such that } \widehat{W}_{\lambda}-\lambda \cdot y \in \hat{H} \\
\int_{\mathscr{Y} \cap\left(\mathbb{R}^{N} \backslash S\right)} A(y) \nabla \widehat{W}_{\lambda} \nabla \varphi=0 \quad \forall \varphi \in \hat{H}
\end{array}\right.
$$

Here, $\hat{H}$ is the space of functions on $\mathscr{Y} \cap\left(\mathbb{R}^{N} \backslash S\right)$ defined by

$$
\hat{H} \doteq\left\{v \in L_{\mathrm{loc}}^{2}\left(\mathscr{Y} \cap\left(\mathbb{R}^{N} \backslash S\right)\right), \nabla v \in L^{2}\left(\mathscr{Y} \cap\left(\mathbb{R}^{N} \backslash S\right)\right), \forall i \in \mathcal{I}, v \in H_{\mathrm{per}}^{1}\left(\mathscr{Y}_{s}^{i}\right), \mathcal{M}_{\mathscr{y} / i}(v)=0\right\}
$$

endowed with the norm

$$
\|v\|_{H}=\|\nabla v\|_{L^{2}\left(\mathscr{Y} \cap\left(\mathbb{R}^{N} \backslash S\right)\right)}
$$

Proof. The function $\widehat{W}_{\lambda}$ is obtained on each connected component of $\mathscr{Y} \cap\left(\mathbb{R}^{N} \backslash S\right)$ by applying Proposition 3.8 . It only remains to check that $\nabla \widehat{W}_{\lambda}$ belongs to $L^{2}\left(\mathscr{Y} \cap\left(\mathbb{R}^{N} \backslash S\right)\right)$. This follows by summing, over $i \in \mathcal{I}$, for $\widehat{\eta}_{\lambda} \doteq \lambda \cdot y-\widehat{W}_{\lambda}$, the following inequality

$$
\alpha^{2} \int_{\mathscr{Y}_{s}^{i}}\left|\nabla \widehat{\eta}_{\lambda}\right|^{2} \leq \int_{\mathscr{Y}_{s}^{i}}|A(y) \lambda|^{2}
$$

which, due to $(6.3)$, is a consequence of

$$
\alpha \int_{\mathscr{Y}_{s}^{i}}\left|\nabla \widehat{\eta}_{\lambda}\right|^{2} \leq \int_{\mathscr{Y}_{s}^{i}} A(y) \nabla \widehat{\eta}_{\lambda} \nabla \widehat{\eta}_{\lambda}=\int_{\mathscr{Y}_{s}^{i}} A(y) \lambda \nabla \widehat{\eta}_{\lambda} .
$$

Corollary 6.2. Problem (4.6) has a unique solution.

The following proposition is one of the main ingredients for the proof of Theorem 5.10.

Proposition 6.3. Let $\widehat{W}_{\lambda}^{\varepsilon}$ be the unique solution of (5.6) and set $\widehat{\eta}_{\lambda}^{\varepsilon}=\lambda \cdot y-\widehat{W}_{\lambda}^{\varepsilon}$, which is the solution of the variational problem

$$
\int_{\mathscr{Y} \varepsilon} A(y) \nabla \widehat{\eta}_{\lambda}^{\varepsilon}(y)=\int_{\mathscr{Y} \varepsilon} A(y) \lambda \nabla \varphi \quad \forall \varphi \in H_{\varepsilon},
$$


where $H_{\varepsilon}$ is defined in (3.15). Under the assumptions of Theorem 5.10, the following convergences hold:

$$
\left\{\begin{array}{l}
{\left[\nabla \widehat{W}_{\lambda}^{\varepsilon}\right] \sim\left[\nabla \widehat{W}_{\lambda}\right] \sim \text { weakly in }\left(L^{2}(\mathscr{Y})\right)^{N},} \\
A\left[\nabla \widehat{W}_{\lambda}^{\varepsilon}\right] \sim A\left[\nabla \widehat{W}_{\lambda}\right] \sim \text { weakly in }\left(L^{2}(\mathscr{Y})\right)^{N}, \\
\varepsilon\left[\widehat{\eta_{\lambda}^{\varepsilon}}\right] \sim 0 \text { strongly in } L^{2}(\mathscr{Y}),
\end{array}\right.
$$

where $\widehat{W}_{\lambda}$ is the unique solution of (5.6) and

$$
\left[\nabla \widehat{W}_{\lambda}^{\varepsilon}\right] \sim=\left\{\begin{array}{l}
\nabla \widehat{W}_{\lambda}^{\varepsilon} \text { on } \mathscr{Y}_{\varepsilon} \\
0 \quad \text { on } \mathcal{T}_{\varepsilon}
\end{array}, \quad\left[\nabla \widehat{W}_{\lambda}\right] \sim=\left\{\begin{array}{ll}
\nabla \widehat{W}_{\lambda} & \text { on } \mathscr{Y} / 0 \\
0 & \text { on } \mathcal{T}_{0}
\end{array}, \quad\left[\widehat{\eta}_{\lambda}^{\varepsilon}\right] \sim= \begin{cases}\eta_{\lambda}^{\varepsilon} & \text { on } \mathscr{Y} \varepsilon \\
0 & \text { on } \mathcal{T}_{\varepsilon} .\end{cases}\right.\right.
$$

Moreover,

$$
\left\{\begin{array}{l}
{\left[\nabla \widehat{w}_{\lambda}^{\varepsilon}\right]^{\sim} \rightarrow \frac{|\mathscr{Q}|}{|\mathscr{Y}|} \mathcal{M}_{\mathscr{Y} / 0}\left(\nabla \widehat{W}_{\lambda}\right) \quad \text { weakly in }\left(L^{2}(\Omega)\right)^{N},} \\
A^{\varepsilon}\left[\nabla \widehat{w}_{\lambda}^{\varepsilon}\right]^{\sim} \rightarrow \frac{|\mathscr{Q}|}{|\mathscr{Y}|} \mathcal{M}_{\mathscr{Y} 0}\left(A \nabla \widehat{W}_{\lambda}\right) \quad \text { weakly in }\left(L^{2}(\Omega)\right)^{N}, \\
\varepsilon\left[\widehat{\eta_{\lambda}^{\varepsilon}(\dot{\bar{\varepsilon}})}\right]^{\sim} \rightarrow 0 \quad \text { strongly in } L^{2}(\Omega), \\
{\left[\widehat{w}_{\lambda}^{\varepsilon}\right]^{\sim} \rightarrow \theta(\lambda \cdot x) \quad \text { weakly in } L^{2}(\Omega),}
\end{array}\right.
$$

where

$$
\widehat{w}_{\lambda}^{\varepsilon}(x)=\varepsilon \widehat{W}_{\lambda}^{\varepsilon}\left(\frac{x}{\varepsilon}\right), \quad \text { on } \Omega_{\varepsilon} .
$$

In the case of cracks, $\theta=1$, the zero extensions in (6.5) and (6.6) are not necessary and the last convergence in (6.6) is strong.

Proof. The existence of $\widehat{W}_{\lambda}$ is given by Corollary 6.2. As in the proof of Proposition 3.10, the convergences in (6.6) follow directly from Proposition 3.1 and the convergences of (6.5), which we now establish.

By the choice $\varphi=\widehat{\eta}_{\lambda}^{\varepsilon}$ in (6.4) and by (2.5), one concludes that

$$
\left\|\nabla \widehat{\eta}_{\lambda}^{\varepsilon}\right\|_{L^{2}(\mathscr{H} \varepsilon)} \leq C, \quad\left\|\nabla \widehat{W}_{\lambda}^{\varepsilon}\right\|_{L^{2}(\mathscr{H} \varepsilon)} \leq C
$$

Consequently, up to a subsequence, we can assume that

$$
\left[\nabla \widehat{W}_{\lambda}^{\varepsilon}\right] \sim-\sigma_{\lambda} \text { weakly in }\left(L^{2}(\mathscr{Y})\right)^{N}
$$

Let $\varphi$ be a smooth $\mathscr{Y} 0$-periodic function and let $\varphi_{\varepsilon} \in H_{\text {per }}^{1}\left(\mathscr{Y}_{\varepsilon}\right)$ be the function given by Assumption 5.6. Then

$$
0=\int_{\mathscr{Y} / \varepsilon} A(y) \nabla \widehat{W}_{\lambda}^{\varepsilon}(y) \nabla \varphi_{\varepsilon}(y) \mathrm{d} y=\int_{\mathscr{Y}} \chi_{\mathscr{Y} / \varepsilon} A(y)\left[\nabla \widehat{W}_{\lambda}^{\varepsilon}\right] \sim(y)\left[\nabla \varphi_{\varepsilon}\right]^{\sim}(y) \mathrm{d} y
$$

Passing to the limit as $\varepsilon \rightarrow 0$ while using (6.8) and Assumptions 5.4i) and 4.6, yields

$$
0=\int_{\mathscr{Y}} \chi_{\mathscr{Y} / 0} A(y) \sigma_{\lambda}(y) \nabla \varphi(y) \mathrm{d} y=\int_{\mathscr{Y} / 0} A(y) \sigma_{\lambda}(y) \nabla \varphi(y) \mathrm{d} y .
$$

This means that

$$
-\operatorname{div}\left(A \sigma_{\lambda}\right)=0 \quad \text { in } \mathscr{Y} 0
$$


Convergence i) of Assumption 5.4 implies that $\sigma_{\lambda}=0$ in $\mathcal{T}_{0}$. Then, to prove the first two convergences it suffices to shows that $\sigma_{\lambda}=\nabla \widehat{W}_{\lambda}$ on $\mathscr{Y}_{0}$. By uniqueness of the solution of (5.6), it is enough to establish that $\sigma_{\lambda}$ is a gradient on $\mathscr{Y} 0$.

To do so, we apply the De Rham's theorem, which states that $\sigma_{\lambda}$ is a gradient on $\mathscr{H}_{0}$ if

$$
\int_{\mathscr{Y} / 0} \sigma_{\lambda} \cdot g \mathrm{~d} y=0
$$

for all divergence-free $g \in\left(\mathcal{D}\left(\mathscr{Y}_{0}^{i}\right)\right)^{N}$, for every $i \in \mathcal{I}$. Indeed, from Assumption 5.4ii), such a $g$ is also in $(\mathcal{D}(\mathscr{Y} \varepsilon))^{\mathcal{N}}$, for $\varepsilon$ small enough, so that we have

$$
0=\int_{\mathscr{Y} / \varepsilon} \nabla \widehat{W}_{\lambda}^{\varepsilon} \cdot g \mathrm{~d} y=\int_{\mathscr{Y}} \chi_{\mathscr{H} \varepsilon}\left[\nabla \widehat{W}_{\lambda}^{\varepsilon}\right] \sim \cdot g \mathrm{~d} y \rightarrow \int_{\mathscr{Y}} \chi_{\mathscr{Y} / 0} \sigma_{\lambda} \cdot g \mathrm{~d} y=\int_{\mathscr{Y} / 0} \sigma_{\lambda} \cdot g \mathrm{~d} y=0 .
$$

Finally, the third convergence concerning $\widehat{\eta}_{\lambda}^{\varepsilon}$ follows directly from (6.7) together with Assumption 5.4i).

The next essential tool for proving Theorem 5.10 is a compactness result in $L^{2}$. This kind of result was originally introduced in [2] in the case of a fixed reference hole, making use of the Kolmogorov compactness criterion. A variant of this result was given in [7] (Lem. 4.1), in a particular geometrical situation of varying reference holes. The proof makes use of a singular perturbation argument, which actually applies in our context. We give it here for the reader's convenience.

Proposition 6.4. Under Assumption 5.1, let $\Omega_{\varepsilon}$ be defined by (2.6). Suppose that for every $\varepsilon$, the domain $\mathscr{Y}_{\varepsilon}$ satisfies the Poincaré-Wirtinger inequality and that (cf. (5.4))

$$
\lim _{\varepsilon \rightarrow 0} \varepsilon C(\mathscr{Y} \varepsilon)=0 .
$$

Let $\left\{u_{\varepsilon}\right\}_{\varepsilon}$ be a sequence in $L^{2}(\Omega)$ with $u_{\varepsilon}=0$ on $\Omega \backslash \Omega_{\varepsilon}$ and $u_{\varepsilon \mid \Omega_{\varepsilon}}$ in $H^{1}\left(\Omega_{\varepsilon}\right)$ for every $\varepsilon$, such that

$$
\left\|\nabla u_{\varepsilon}\right\|_{L^{2}\left(\Omega_{\varepsilon}\right)} \text { is bounded and } u_{\varepsilon} \rightarrow u_{0} \text { weakly in } L^{2}(\Omega) .
$$

Let $\left\{K_{\varepsilon}\right\}_{\varepsilon}$ be a bounded sequence in $L_{\mathrm{per}}^{2}(\mathscr{Y})$ such that

$$
\mathcal{M}_{\mathscr{Y} \varepsilon}\left(K_{\varepsilon}\right) \rightarrow K_{0} \in \mathbb{R}
$$

Then

$$
\forall \varphi \in \mathcal{D}(\Omega), \quad \int_{\Omega} K_{\varepsilon}\left(\frac{x}{\varepsilon}\right) u_{\varepsilon}(x) \varphi(x) \mathrm{d} x \rightarrow K_{0} \int_{\Omega} u_{0}(x) \varphi(x) \mathrm{d} x .
$$

Proof. Suppose first that $\operatorname{Int}\left(\mathcal{T}_{\varepsilon}\right) \neq \emptyset$. From (6.10) and (6.11) one has

$$
\forall \varphi \in \mathcal{D}^{\prime}(\Omega), \quad \int_{\Omega} \mathcal{M}_{\mathscr{Y} \varepsilon}\left(K_{\varepsilon}\right) u_{\varepsilon}(x) \varphi(x) \mathrm{d} x \rightarrow K_{0} \int_{\Omega} u_{0}(x) \varphi(x) \mathrm{d} x .
$$

It remains to prove (6.12) with zero right-hand side, for the sequence $\left\{Z_{\varepsilon}\right\}_{\varepsilon}$, instead of $\left\{K_{\varepsilon}\right\}_{\varepsilon}$, where $Z_{\varepsilon}=$ $K_{\varepsilon}-\mathcal{M}_{\mathscr{Q} \varepsilon}\left(K_{\varepsilon}\right)$ has the extra property $\mathcal{M}_{\mathscr{Y} \varepsilon}\left(Z_{\varepsilon}\right)=0$.

Let $V_{\varepsilon} \in H_{\mathrm{per}}^{1}\left(\mathscr{Y}_{\varepsilon}\right)$ be the solution, in the variational sense, of

$$
\left\{\begin{array}{l}
-\frac{1}{\varepsilon^{2}} \Delta V_{\varepsilon}+V_{\varepsilon}=Z_{\varepsilon} \quad \text { in } \mathscr{Y} \varepsilon \\
\frac{\partial V_{\varepsilon}}{\partial n}=0 \text { on } \partial \mathcal{T}_{\varepsilon}, \\
V_{\varepsilon} \quad \mathscr{Y} \text { - periodic. }
\end{array}\right.
$$


By using the function 1 as test in (6.13), one deduces that $\mathcal{M}_{\mathscr{Y} \varepsilon}\left(V_{\varepsilon}\right)=\mathcal{M}_{\mathscr{Y} \varepsilon}\left(Z_{\varepsilon}\right)=0$. By taking $V_{\varepsilon}$ as test, one obtains

$$
\frac{1}{\varepsilon^{2}} \int_{\mathscr{Y} \varepsilon}\left|\nabla V_{\varepsilon}\right|^{2}+\int_{\mathscr{Y} \varepsilon} V_{\varepsilon}^{2}=\int_{\mathscr{Y} \varepsilon} V_{\varepsilon} Z_{\varepsilon}
$$

Since $\left\{Z_{\varepsilon}\right\}_{\varepsilon}$ is bounded in $L^{2}(\mathscr{H})$ by some $M$, one deduces the following estimates:

$$
\left\|\nabla V_{\varepsilon}\right\|_{L^{2}(\mathscr{Y} \varepsilon)} \leq M \varepsilon \quad \text { and } \quad\left\|V_{\varepsilon}\right\|_{L^{2}(\mathscr{Y} \varepsilon)} \leq M
$$

Now, it follows from (5.4) that

$$
\left\|V_{\varepsilon}\right\|_{L^{2}(\mathscr{Y} \varepsilon)}=\left\|V_{\varepsilon}-\mathcal{M}_{\mathscr{Y} \varepsilon}\left(V_{\varepsilon}\right)\right\|_{L^{2}(\mathscr{Y} \varepsilon)} \leq M \varepsilon C\left(\mathscr{Y}_{\varepsilon}\right) \rightarrow 0
$$

This, together with (6.14) yields

$$
\frac{1}{\varepsilon^{2}} \int_{\mathscr{Y} \varepsilon}\left|\nabla V_{\varepsilon}\right|^{2}+\int_{\mathscr{Y} \varepsilon} V_{\varepsilon}^{2} \rightarrow 0
$$

Set $v_{\varepsilon}(x)=V_{\varepsilon}\left(\frac{x}{\varepsilon}\right)$, which is $\varepsilon \mathscr{Z}$-periodic and from (6.13) satisfies

$$
\left\{\begin{array}{l}
-\Delta v_{\varepsilon}+v_{\varepsilon}=Z_{\varepsilon}\left(\frac{x}{\varepsilon}\right) \quad \text { in } \mathbb{R}^{N} \backslash T_{\varepsilon}\left(\mathbb{R}^{N}, \mathcal{T}_{\varepsilon}\right) \\
\frac{\partial v_{\varepsilon}}{\partial n}=0 \quad \text { on } \partial T_{\varepsilon}\left(\mathbb{R}^{N}, \mathcal{T}_{\varepsilon}\right)
\end{array}\right.
$$

From (6.15), for every bounded open set $\omega$ of $\mathbb{R}^{N}$, one has,

$$
\int_{\omega \cap\left(\mathbb{R}^{N} \backslash T_{\varepsilon}\left(\mathbb{R}^{N}, \mathcal{T}_{\varepsilon}\right)\right)}\left(\left|\nabla v_{\varepsilon}\right|^{2}+v_{\varepsilon}^{2}\right) \rightarrow 0
$$

For any $\varphi \in \mathcal{D}(\Omega)$, using $\varphi u_{\varepsilon}$ as test in (6.16) and since $u_{\varepsilon}$ vanishes outside $\Omega_{\varepsilon}$, one has

$$
\left.\int_{\Omega} Z_{\varepsilon}\left(\frac{x}{\varepsilon}\right) \varphi(x) u_{\varepsilon}(x) \mathrm{d} x=\int_{\Omega_{\varepsilon}} \nabla v_{\varepsilon} \nabla\left(\varphi u_{\varepsilon}\right)+v_{\varepsilon} \varphi u_{\varepsilon}=\int_{\omega \cap\left(\mathbb{R}^{N} \backslash T_{\varepsilon}\left(\mathbb{R}^{N}, \mathcal{T}_{\varepsilon}\right)\right)} \nabla u_{\varepsilon}\right)+v_{\varepsilon} \varphi u_{\varepsilon}
$$

where we have set $\omega=\operatorname{Int}(\operatorname{supp}(\varphi))$ and used the fact that $\Omega_{\varepsilon} \cap \omega=\omega \cap\left(\mathbb{R}^{N} \backslash T_{\varepsilon}\left(\mathbb{R}^{N}, \mathcal{T}_{\varepsilon}\right)\right)$ for $\varepsilon$ sufficiently small.

One easily concludes using $(6.10,6.17)$ and the Cauchy-Schwarz inequality.

The case where $\mathcal{T}_{\varepsilon}$ has zero Lebesgue measure is proved in a similar way, taking into account the fact that $\Omega \backslash \Omega_{\varepsilon}$ is of zero Lebesgue-measure.

Proof of Theorem 5.10. Once Propositions 6.1, 6.3 and 6.4 are established, the proof follows along the lines of the well-know Tartar method [30] of oscillating test functions $w_{\lambda}^{\varepsilon}$, constructed here as follows.

For every $\lambda \in \mathbb{R}^{N}$, let $W_{\lambda}$ be the unique solution of the problem

$$
\left\{\begin{array}{l}
-\operatorname{div}\left({ }^{t} A(y) \nabla W_{\lambda}\right)=0 \quad \text { in } \mathscr{Y}_{0}, \\
\left({ }^{t} A(y) \nabla W_{\lambda}\right) \cdot \nu=0 \quad \text { on } \partial \mathcal{T}_{0}, \\
W_{\lambda}-\lambda \cdot y \quad \mathscr{Y}-\text { periodic }, \\
\mathcal{M}_{\mathscr{Y}_{0}^{i}}\left(W_{\lambda}-\lambda \cdot y\right)=0, \quad i \in \mathcal{I} .
\end{array}\right.
$$

Set

$$
w_{\lambda}^{\varepsilon}(x)=\varepsilon W_{\lambda}^{\varepsilon}\left(\frac{x}{\varepsilon}\right), \eta_{\lambda}^{\varepsilon}=\lambda \cdot y-W_{\lambda}^{\varepsilon} \quad \text { on } \Omega_{\varepsilon}
$$


Proposition 6.3 apply here (with ${ }^{t} A$ in place of $A$ ) so that the following convergences hold:

$$
\left\{\begin{array}{l}
{\left[\nabla W_{\lambda}^{\varepsilon}\right]^{\sim} \rightarrow\left[\nabla W_{\lambda}\right]^{\sim} \quad \text { weakly in }\left(L^{2}(\mathscr{Y})\right)^{N},} \\
{ }^{t} A\left[\nabla W_{\lambda}^{\varepsilon}\right]^{\sim} \rightarrow{ }^{t} A\left[\nabla_{\lambda}\right]^{\sim} \quad \text { weakly in }\left(L^{2}(\mathscr{Y})\right)^{N}, \\
\varepsilon\left[\eta_{\lambda}^{\varepsilon}\right]^{\sim} \rightarrow 0 \quad \text { strongly in } L^{2}(\mathscr{Y}) .
\end{array}\right.
$$

Here, as well as in the remainder of the proof, there is no need to use the zero extension if $\mathcal{T}_{\varepsilon}$ has zero Lebesgue measure.

From (5.3), by weak compactness in $L^{2}(\Omega)$, there exists a subsequence (still denoted $\varepsilon$ ), such that

$$
\left\{\begin{array}{lc}
i . & \widetilde{u_{\varepsilon}} \rightarrow \theta u \quad \text { weakly in } L^{2}(\Omega), \\
i i . & \xi^{\varepsilon} \rightarrow \xi^{0} \quad \text { weakly in }\left(L^{2}(\Omega)\right)^{N}
\end{array}\right.
$$

where

and satisfies

$$
\xi^{\varepsilon}=\left[A^{\varepsilon} \nabla u^{\varepsilon}\right]^{\sim}
$$

$$
\int_{\Omega} \xi^{\varepsilon} \nabla v+\int_{\Omega} a_{0}^{\varepsilon} \widetilde{u_{\varepsilon}} v=\int_{\Omega} \chi_{\Omega_{\varepsilon}} f v, \quad \forall v \in H_{0}^{1}(\Omega) .
$$

From Proposition 6.4, applied to $K_{\varepsilon}=a_{0}$ and $\varphi=v \in \mathcal{D}(\Omega)$ one has

$$
\int_{\Omega} a_{0}^{\varepsilon} \widetilde{u_{\varepsilon}} v \rightarrow \mathcal{M}_{\mathscr{Y} 0}\left(a_{0}\right) \int_{\Omega} \theta u v, \quad \forall v \in \mathcal{D}(\Omega),
$$

where we used Assumption 5.4i). Hence, $\xi^{0}$ satisfies

$$
\int_{\Omega} \xi^{0} \nabla v+\theta \mathcal{M}_{\mathscr{Y} 0}\left(a_{0}\right) \int_{\Omega} u v=\theta \int_{\Omega} f v, \quad \forall v \in H_{0}^{1}(\Omega),
$$

i.e.

$$
-\operatorname{div} \xi^{0}+\theta \mathcal{M}_{\mathscr{Y} 0}\left(a_{0}\right) u=\theta f \quad \text { in } \Omega .
$$

Therefore, the result is proved if we show that

$$
\xi^{0}=A^{0} \nabla u
$$

Indeed, this implies that $u$ belongs to $H^{1}(\Omega)$ since $A^{0}$ is not singular. Hence, due to (6.24), $u$ satisfies (5.8).

Let $\varphi \in \mathcal{D}(\Omega)$ and choose $\varphi w_{\lambda}^{\varepsilon}$ as test function in (6.22) and $\varphi u^{\varepsilon}$ as test function in (6.6). We have respectively,

$$
\begin{aligned}
& \int_{\Omega_{\varepsilon}} \xi^{\varepsilon} \cdot \nabla w_{\lambda}^{\varepsilon} \varphi+\int_{\Omega_{\varepsilon}} \xi^{\varepsilon} \cdot \nabla \varphi w_{\lambda}^{\varepsilon}+\int_{\Omega_{\varepsilon}} a_{0}^{\varepsilon} u_{\varepsilon} \varphi w_{\lambda}^{\varepsilon}=\int_{\Omega_{\varepsilon}} \chi_{\Omega_{\varepsilon}} f \varphi w_{\lambda}^{\varepsilon}, \quad \forall \varphi \in \mathcal{D}(\Omega), \\
& \int_{\Omega_{\varepsilon}}{ }^{t} A \nabla w_{\lambda}^{\varepsilon} \cdot \nabla u^{\varepsilon} \varphi \mathrm{d} x+\int_{\Omega_{\varepsilon}}{ }^{t} A \nabla w_{\lambda}^{\varepsilon} \cdot \nabla \varphi u^{\varepsilon} \mathrm{d} x=0, \quad \forall \varphi \in \mathcal{D}(\Omega) .
\end{aligned}
$$

Observe that by definition

$$
\xi^{\varepsilon} \cdot \nabla w_{\lambda}^{\varepsilon}=A^{\varepsilon} \nabla u^{\varepsilon} \cdot \nabla w_{\lambda}^{\varepsilon} \quad \text { in } \Omega_{\varepsilon} .
$$

Therefore by subtraction

$$
\int_{\Omega} \xi^{\varepsilon} \cdot \nabla \varphi\left[w_{\lambda}^{\varepsilon}\right]^{\sim}-\int_{\Omega}{ }^{t} A\left[\nabla w_{\lambda}^{\varepsilon}\right]^{\sim} \cdot \nabla \varphi u^{\varepsilon}+\int_{\Omega} a_{0}^{\varepsilon} \widetilde{u_{\varepsilon}} \varphi\left[w_{\lambda}^{\varepsilon}\right]^{\sim}=\int_{\Omega} \chi_{\Omega_{\varepsilon}} f \varphi\left[w_{\lambda}^{\varepsilon}\right]^{\sim} .
$$


From (6.20) and (6.21) we have

$$
\begin{gathered}
\lim _{\varepsilon \rightarrow 0} \int_{\Omega} \xi^{\varepsilon} \cdot \nabla \varphi\left[w_{\lambda}^{\varepsilon}\right]^{\sim}=\lim _{\varepsilon \rightarrow 0} \int_{\Omega}\left(\xi^{\varepsilon} \cdot \nabla \varphi\right)\left((\lambda \cdot x)-\varepsilon\left[\widehat{\eta_{\lambda}^{\varepsilon}(\dot{\bar{\varepsilon}})}\right] \sim\right) \\
=\lim _{\varepsilon \rightarrow 0} \int_{\Omega}\left(\xi^{\varepsilon} \cdot \nabla \varphi\right)(\lambda \cdot x)=\int_{\Omega} \xi^{0} \cdot \nabla \varphi(\lambda \cdot x) .
\end{gathered}
$$

Similarly,

$$
\lim _{\varepsilon \rightarrow 0} \int_{\Omega} \chi_{\Omega_{\varepsilon}} f \varphi\left[w_{\lambda}^{\varepsilon}\right]^{\sim}=\lim _{\varepsilon \rightarrow 0} \int_{\Omega} \chi_{\Omega_{\varepsilon}} f \varphi\left((\lambda \cdot x)-\varepsilon\left[\widehat{\eta_{\lambda}^{\varepsilon}(\dot{\bar{c}})}\right] \sim\right)=\theta \int_{\Omega} f \varphi(\lambda \cdot x) .
$$

On the other hand

$$
\lim _{\varepsilon \rightarrow 0} \int_{\Omega} a_{0}^{\varepsilon} \widetilde{u_{\varepsilon}} \varphi\left[w_{\lambda}^{\varepsilon}\right]^{\sim}=\lim _{\varepsilon \rightarrow 0} \int_{\Omega} a_{0}^{\varepsilon} \widetilde{u_{\varepsilon}} \varphi\left((\lambda \cdot x)-\varepsilon\left[\widehat{\eta_{\lambda}^{\varepsilon}\left(\dot{\frac{c}{\varepsilon}}\right)}\right] \sim\right)=\lim _{\varepsilon \rightarrow 0} \int_{\Omega} a_{0}^{\varepsilon} \widetilde{u_{\varepsilon}} \varphi(\lambda \cdot x) .
$$

The same argument used to prove (6.24) (applying Prop. 6.4) gives

$$
\lim _{\varepsilon \rightarrow 0} \int_{\Omega} a_{0}^{\varepsilon} \widetilde{u_{\varepsilon}} \varphi\left[w_{\lambda}^{\varepsilon}\right]^{\sim}=\theta \mathcal{M}_{\mathscr{H}_{0}}\left(a_{0}\right) \int_{\Omega} u \varphi(\lambda \cdot x) .
$$

Finally, from (6.20) and Proposition 6.4, applied to $K_{\varepsilon}={ }^{t} A\left[\nabla W_{\lambda}^{\varepsilon}\right]^{\sim}$ and $\nabla \varphi$ one has

$$
\lim _{\varepsilon \rightarrow 0} \int_{\Omega}{ }^{t} A\left[\nabla w_{\lambda}^{\varepsilon}\right]^{\sim} \cdot \nabla \varphi u^{\varepsilon}=\theta \int_{\Omega} B^{0} \lambda \cdot \nabla \varphi u,
$$

where $B^{0}$ is defined by $B^{0} \lambda=\mathcal{M}_{\mathscr{Y}}\left({ }^{t} A\left[\nabla W_{\lambda}\right]^{\sim}\right)$.

Then, passing to the limit in $(6.26)$ yields

$$
\int_{\Omega} \xi^{0} \cdot \nabla \varphi(\lambda \cdot x)-\int_{\Omega} B^{0} \lambda \cdot \nabla \varphi u+\theta \mathcal{M}_{\mathscr{Y}_{0}}\left(a_{0}\right) \int_{\Omega} u \varphi(\lambda \cdot x)=\theta \int_{\Omega} f \varphi(\lambda \cdot x) .
$$

This gives, together with (6.24), written for $v=(\lambda \cdot x) \varphi$,

$$
\int_{\Omega} \xi^{0} \cdot \lambda \varphi d x=-\int_{\Omega} B^{0} \lambda \cdot \nabla \varphi u^{0} \quad \forall \varphi \in \mathcal{D}(\Omega),
$$

which implies that

since $\lambda$ is arbitrary in $\mathbb{R}^{N}$.

$$
\xi^{0}={ }^{t} B^{0} \nabla u, \quad \text { a.e. }
$$

It remains to prove that $A^{0}={ }^{t} B^{0}$, i.e.,

$$
B^{0} \lambda \cdot \mu=A^{0} \mu \cdot \lambda, \quad \forall \lambda, \mu \in \mathbb{R}^{N} .
$$

From the definition of $B^{0}$ one has

$$
B^{0} \lambda \cdot \mu=\frac{1}{|\mathscr{Y}|} \int_{\mathscr{Y} / 0}^{t} A(y)\left(\lambda-\nabla \eta_{\lambda}\right) \mu \mathrm{d} y=\frac{1}{|\mathscr{Y}|} \int_{\mathscr{H} 0} A(y) \mu \cdot \lambda \mathrm{d} y-\frac{1}{|\mathscr{Y}|} \int_{\mathscr{Y} / 0} A(y) \mu \nabla \eta_{\lambda} \mathrm{d} y .
$$

From (5.10) we obtain

$$
\int_{\mathscr{H}_{0}} A(y) \nabla \widehat{\eta}_{\mu} \nabla \varphi \mathrm{d} y=\int_{\mathscr{H}_{0}} A(y) \mu \nabla \varphi \mathrm{d} y \quad \forall \varphi \in H
$$


By choosing $\varphi=\eta_{\lambda}$ in $(6.27)$, one has

$$
B^{0} \lambda \cdot \mu=\frac{1}{|\mathscr{Y}|} \int_{\mathscr{Y} 0} A(y) \mu \cdot \lambda \mathrm{d} y-\frac{1}{|\mathscr{Y}|} \int_{\mathscr{Y}_{0}} A(y) \nabla \widehat{\eta}_{\mu} \nabla \eta_{\lambda} \mathrm{d} y
$$

A similar computation starting with the definition of $A^{0}$, gives

$$
A^{0} \mu \cdot \lambda=\frac{1}{|\mathscr{Y}|} \int_{\mathscr{Y} 0}{ }^{t} A(y) \lambda \cdot \mu \mathrm{d} y-\frac{1}{|\mathscr{Y}|} \int_{\mathscr{H} 0}{ }^{t} A(y) \nabla \eta_{\lambda} \nabla \widehat{\eta}_{\mu} \mathrm{d} y,
$$

which ends the proof in the case where $\operatorname{Int}\left(\mathcal{T}_{\varepsilon}\right) \neq \emptyset$. Finally, the proof of (5.9) follows that of Attouch-Murat (see [3]), which extends to our case by making use of Assumption 5.4ii).

Remark 6.5. When the reference hole is independent of $\varepsilon\left(\right.$ i.e. $\mathcal{T}_{\varepsilon} \equiv \mathcal{T}$ ), another approach to these type of problems is presented in several papers of Zhikov (see [33]), which introduce the notion of "2-connectedness" and allows for some disconnected $\mathscr{Y} \backslash \mathcal{T}$ but with connected closure. The Sobolev space used there is the closure of $C^{\infty}\left(\overline{\mathscr{Y} \backslash \mathcal{T})}\right.$ for the $H^{1}$-norm, which is smaller than the space used here. The analogous of Proposition 6.4 of the present paper is proved by a property of the heat semi-group (Prop. 9.4 of [33]). It is not clear whether there exist conditions (more general than our (5.4)) which guarantee a uniform version of this heat semi-group property for $\varepsilon$-dependent reference holes.

\section{Domains for Which the Poincaré-Wirtinger Property holds}

One of the main assumption in Theorem 5.10 (and in the related results) is the Poincaré-Wirtinger property (PWI) given in Definition 3.3. In this paragraph we summarize general results connecting it with some geometric properties of a bounded domain $\mathcal{O}$ of $R^{N}$, in the same spirit as in Section 4.

Definition 7.1. For $p \in[1, \infty]$, the bounded domain $\mathcal{O}$ in $R^{N}$ is a $p$-Poincaré-Wirtinger domain (in short a $p$-PW domain) whenever the Poincaré-Wirtinger inequality holds for all elements of $W^{1, p}(\mathcal{O})$ (with a constant depending only on $\mathcal{O}$ and $p$ ):

$$
\left\|u-\mathcal{M}_{\mathcal{O}}(u)\right\|_{L^{p}(\mathcal{O})} \leq C(\mathcal{O}, p)\|\nabla u\|_{L^{p}(\mathcal{O})} .
$$

The case for Theorem 5.10 is that of $p=2$.

A variant of this property, related to Sobolev embeddings, is the following:

Definition 7.2. For $p \in[1, N)$, the bounded domain $\mathcal{O}$ in $R^{N}$ is a $p$-Sobolev-Poincaré-Wirtinger domain (in short a $p$-SP domain) whenever the Sobolev-Poincaré-Wirtinger inequality holds for all elements of $W^{1, p}(\mathcal{O})$ with zero average on $\mathcal{O}$ (with a constant depending on $\mathcal{O}$ and $p$ ):

$$
\left\|u-\mathcal{M}_{\mathcal{O}}(u)\right\|_{L^{p^{*}}(\mathcal{O})} \leq C(\mathcal{O}, p)\|\nabla u\|_{L^{p}(O)},
$$

where $p^{*}=\frac{N p}{N-p}$.

It is clear that, for either property to hold, connectedness of $\mathcal{O}$ is necessary. It is also obvious that $p$-SP implies $p$-PW, and it is known that the converse is not true in general.

The same argument used to prove Proposition 3.5 (considered for $S=\emptyset$ ), implies that if for $1<p<\infty$, the embedding from $W^{1, p}(\mathcal{O})$ in $L^{p}(\mathcal{O})$ is compact and $\mathcal{O}$ is connected, then $\mathcal{O}$ is a $p$-PW domain. A similar statement holds for $p$-SP domains.

Consequently, due to Theorem 4.4, Jones-domains (see Sect. 3) are $p$-PW domains for every $p(1<p<\infty)$.

Proposition 7.3. If two intersecting domains $\mathcal{O}_{1}$ and $\mathcal{O}_{2}$, are $p$ - $P W$ domains (resp. p-SP domains), so is their union. 
Proof. By contradiction suppose that $\mathcal{O}=\mathcal{O}_{1} \cup \mathcal{O}_{2}$ is not a $p$-PW domain. Then, there exists a sequence $\left(u_{n}\right)$ in $W^{1, p}(\mathcal{O})$ such that

$$
\begin{cases}\text { i) } & \mathcal{M}_{\mathcal{O}}\left(u_{n}\right)=0, \\ \text { ii }) \quad\left\|u_{n}\right\|_{L^{p}(\mathcal{O})}=1, \\ \text { iii) } \quad\left\|\nabla u_{n}\right\|_{L^{p}(\mathcal{O})} \rightarrow 0 .\end{cases}
$$

From (7.1)ii), $\mathcal{M}_{\mathcal{O}_{1}}\left(u_{n}\right)$ and $\mathcal{M}_{\mathcal{O}_{2}}\left(u_{n}\right)$ are bounded and (up to a subsequence) one can assume that they converge to some $c_{1}$ and $c_{2}$ respectively.

Since $\mathcal{O}_{1}$ and $\mathcal{O}_{2}$ are p-PW domains, convergence (7.1)iii) implies

$$
\left\|u_{n}-\mathcal{M}_{\mathcal{O}_{1}}\left(u_{n}\right)\right\|_{L^{p}\left(\mathcal{O}_{1}\right)} \rightarrow 0, \quad\left\|u_{n}-\mathcal{M}_{\mathcal{O}_{2}}\left(u_{n}\right)\right\|_{L^{p}\left(\mathcal{O}_{2}\right)} \rightarrow 0
$$

Consequently,

$$
u_{n} \rightarrow c_{1} \text { strongly in } L^{p}\left(\mathcal{O}_{1}\right), \quad u_{n} \rightarrow c_{2} \text { strongly in } L^{p}\left(\mathcal{O}_{2}\right)
$$

Since $\mathcal{O}_{1} \cap \mathcal{O}_{2} \neq \emptyset$, one has $c_{1}=c_{2}$ and from (7.1)i), $c_{1}=c_{2}=0$, so that $u_{n}$ strongly converges to zero in $L^{p}(\mathcal{O})$. This contradicts (7.1)ii).

The following very geometric condition was originally introduced in Martio-Sarvas [22] (see also Martio [20], Gehring-Martio [16], Väisalä [31]):

Definition 7.4. A connected domain $\mathcal{O}$ in $\mathbb{R}^{N}$ is an $(\alpha, \beta)$ John-domain $(0<\alpha \leq \beta)$, provided there exist a point $x \in \mathcal{O}$ (denoted a center) and, for every point $y \in \mathcal{O}$ a rectifiable curve in $\mathcal{O}$ joining $x$ to $y$ with length $\ell(\gamma) \leq \beta$, and along which the following holds:

$$
\inf _{z \in \gamma} \frac{d(z, \partial \mathcal{O})}{\ell(\gamma(z, y))} \geq \alpha .
$$

Here, as before, $d$ is the Euclidian distance, and $\ell(\gamma(z, y))$ is the arc length of the part of $\gamma$ which connects $z$ to $y$.

It can be seen that once a center exists, any other point can also be used as a center, but the values of $\alpha$ and $\beta$ may change. This definition, a sort of twisted cone-condition, prevents the presence of external cusps, but allows for some fractal boundaries (for example, the snowflake domain in $\mathbb{R}^{2}$ ). Indeed,

Theorem 7.5 (see Gehring-Osgood [17], Smith-Stegenga [27]). Every bounded ( $\varepsilon, \delta)$ Jones-domain is an ( $\alpha, \beta)$ John-domain for suitable $\alpha$ and $\beta$ depending only upon $\varepsilon$ and $\delta$.

The converse is not true, as we will see further down.

The notion of John-domains provides a quite general class of domains for which condition (5.4) of Theorem 5.10 holds, due to the following results applied in the case $p=2$.

Theorem 7.6 (Martio [21]). Every $(\alpha, \beta)$ John-domain is a $p$-PW domain for every $p$. Furthermore, the best $p$-Poincaré-Wirtinger constant of such a domain is bounded above by an expression involving only $\alpha, \beta, p$ and $N$.

Actually, this result is a consequence of:

Theorem 7.7 (Bojarski [6]). Every $(\alpha, \beta)$ John-domain is a p-SP domain (for every $p \in[1,+\infty)$ ). Furthermore, the best p-Sobolev Poincaré-Wirtinger constant of such a domain is bounded above by an expression involving only $\alpha, \beta, p$ and $N$.

The latter result is quasi-optimal:

Theorem 7.8 (Buckley-Koskela [9]). Suppose that $\mathcal{O}$ is a bounded domain in $\mathbb{R}^{n}$ and that it satisfies a separation hypothesis (explained below). Then $\mathcal{O}$ is a $p$-SP domain (for some $p \in[1, N)$ ) if and only if it is a John-domain (for some values of $\alpha$ and $\beta$ ). 
The separation condition needed here is the following: there is a point $x \in \mathcal{O}$ (a center) and a constant $C_{0}$ such that for each $y \in \mathcal{O}$, there is a curve $\gamma$ from $x$ to $y$ satisfying that for each $z \in \gamma, \gamma(z, y)$ does not intersect the connected component of $x$ in $\mathcal{O} \backslash \partial B_{z}$, where $B_{z}$ denotes the ball of center $z$ and radius $C_{0} d(z, \partial \mathcal{O})$.

All the conditions indicated so far apply for every $p$ indistinctly. There is a generalization of the notion of John-domain which discriminates between values of $p$. We give it here to show how involved the arguments can be.

Definition 7.9 [27]. Let $\eta \geq 1$. A domain $\mathcal{O}$ in $\mathbb{R}^{N}$ is an $(\alpha, \beta) \eta$-John-domain $(0<\alpha \leq \beta)$, provided there exist a point $x \in \mathcal{O}$ (denoted a center) and, for every point $y \in \mathcal{O}$ a rectifiable curve in $\mathcal{O}$ joining $x$ to $y$ with length $\ell(\gamma) \leq \beta$, and along which the following holds:

$$
\inf _{z \in \gamma} \frac{d(z, \partial \mathcal{O})}{\ell\left(\gamma(z, y)^{\eta}\right.} \geq \alpha .
$$

The corresponding result is:

Theorem 7.10 [27]. Let $1 \leq p<\infty$. If $\mathcal{O} \subset \mathbb{R}^{N}$ is an $\eta$-John-domain with $1 \leq \eta<1+\frac{p}{N-1}$, then $\mathcal{O}$ is a $p$-Poincaré domain. In particular this hold for every $p$ when $\eta<\frac{N}{N-1}$.

To close this section, we indicate that there exists an example in dimension 3 of a 2-Poincaré-Wirtinger domain $\mathcal{O}$ (satisfying the separation hypothesis) for which the embedding from $H^{1}(\mathcal{O})$ to $L^{2}(\mathcal{O})$ is not compact. This precludes that $\mathcal{O}$ have the 2-extension property, hence is an example of a John-domain which is not a Jonesdomain! For this, we refer to [27] where the construction of $\mathcal{O}$ is done by adding to the unit ball a suitable sequence of "rooms" and "corridors" with size converging to zero.

\section{Appendix}

We give here the proof of Proposition 5.13.

Lemma A.1. Under Assumption 5.12, for all $v \in H^{1}\left(\mathscr{Y}_{\varepsilon} \cup \tau_{b_{j}}\left(\mathscr{H}_{\varepsilon}\right)\right)$, one has:

$$
\left\|\tau_{b_{j}}\left(\left.v\right|_{\mathscr{Y} \varepsilon}\right)-\left.v\right|_{\mathscr{Y} \varepsilon}\right\|_{L^{2}(\mathscr{Y} \varepsilon)} \leq \sqrt{2} C^{\prime}\left\|\nabla v_{\varepsilon}\right\|_{L^{2}\left(\mathscr{Y} \varepsilon \cup \tau_{b_{j}}(\mathscr{Y} \varepsilon)\right)}
$$

Proof. We have

$$
\begin{aligned}
& \left\|\tau_{b_{j}}\left(\left.v\right|_{\mathscr{V}_{\varepsilon}}\right)-\left.v\right|_{\mathscr{Y}_{\varepsilon}}\right\|_{L^{2}\left(\mathscr{Y}_{\varepsilon}\right)} \leq\left\|\tau_{b_{j}}\left(\left.v\right|_{\mathscr{H}_{\varepsilon}}\right)-\mathcal{M}_{\mathscr{Y}_{j}^{+}}(v)\right\|_{L^{2}\left(\mathscr{Y}_{\varepsilon}\right)}+\left\|\mathcal{M}_{\mathscr{Y}_{j}^{+}}(v)-\left.v\right|_{\mathscr{Y}_{\varepsilon}}\right\|_{L^{2}\left(\mathscr{Y}_{\varepsilon}\right)} \\
& \leq C^{\prime}\left\|\nabla v_{\varepsilon}\right\|_{L^{2}\left(\tau_{b_{j}}(\mathscr{Y} \varepsilon)\right)}+C^{\prime}\left\|\nabla v_{\varepsilon}\right\|_{L^{2}(\mathscr{Y} \varepsilon)}
\end{aligned}
$$

Proof of Proposition 5.13. Without loss of generality, one can assume that

$$
\widetilde{v}_{\varepsilon} \rightarrow v \quad \text { weakly in } L^{2}(\Omega)
$$

Due to the regularity of the boundary, it is enough to show that the extension by zero of $v$ to $\mathbb{R}^{N}$ is in $H^{1}\left(\mathbb{R}^{N}\right)$.

To do so, we use the classical characterization of $H^{1}\left(\mathbb{R}^{N}\right)$ as the set of the elements $v$ of $L^{2}\left(\mathbb{R}^{N}\right)$ such that for same constant $C$,

for every $h \in \mathbb{R}^{N} \backslash\{0\}$, where

$$
\frac{1}{|h|}\left\|\tau_{h}(v)-v\right\|_{L^{2}\left(\mathbb{R}^{N}\right)} \leq C
$$

$$
\tau_{h}(v)(x) \doteq v(x-h)
$$


Actually, in this characterization, it suffices to take $h$ of the form

$$
h=l b_{j}, j=1, \ldots, N
$$

where $l$ is arbitrary in $\mathbb{R}$. Set $l=\varepsilon \kappa_{\varepsilon}+r_{\varepsilon}$, with $\kappa_{\varepsilon} \in \mathbb{Z}, 0 \leq r_{\varepsilon}<\varepsilon$ and write:

$$
\left\|\tau_{\ell b_{j}}\left(\widetilde{v}_{\varepsilon}\right)-\widetilde{v}_{\varepsilon}\right\|_{L^{2}\left(\mathbb{R}^{N}\right)} \leq\left\|\tau_{\varepsilon \kappa_{\varepsilon} b_{j}}\left(\widetilde{v}_{\varepsilon}\right)-\widetilde{v}_{\varepsilon}\right\|_{L^{2}\left(\mathbb{R}^{N}\right)}+\left\|\tau_{r_{\varepsilon} b_{j}}\left(\widetilde{v}_{\varepsilon}\right)-\widetilde{v}_{\varepsilon}\right\|_{L^{2}\left(\mathbb{R}^{N}\right)}
$$

since the second term of the right hand side equals $\left\|\tau_{\ell b_{j}}\left(\widetilde{v}_{\varepsilon}\right)-\tau_{\varepsilon \kappa_{\varepsilon} b_{j}}\left(\widetilde{v}_{\varepsilon}\right)\right\|_{L^{2}\left(\mathbb{R}^{N}\right)}$, due to the translation invariance of the norm. Similarly,

$$
\left\|\tau_{\varepsilon \kappa_{\varepsilon} b_{j}}\left(\widetilde{v}_{\varepsilon}\right)-\widetilde{v}_{\varepsilon}\right\|_{L^{2}\left(\mathbb{R}^{N}\right)} \leq \sum_{t=1}^{\kappa_{\varepsilon}}\left\|\tau_{\varepsilon t b_{j}}\left(\widetilde{v}_{\varepsilon}\right)-\tau_{\varepsilon(t-1) b_{j}}\left(\widetilde{v}_{\varepsilon}\right)\right\|_{L^{2}\left(\mathbb{R}^{N}\right)} \leq \kappa_{\varepsilon}\left\|\tau_{\varepsilon b_{j}}\left(\widetilde{v}_{\varepsilon}\right)-\widetilde{v}_{\varepsilon}\right\|_{L^{2}\left(\mathbb{R}^{N}\right)}
$$

so that

$$
\left\|\tau_{\ell b_{j}}\left(\widetilde{v}_{\varepsilon}\right)-\widetilde{v}_{\varepsilon}\right\|_{L^{2}\left(\mathbb{R}^{N}\right)} \leq\left|\kappa_{\varepsilon}\right|\left\|\tau_{\varepsilon b_{j}}\left(\widetilde{v}_{\varepsilon}\right)-\widetilde{v}_{\varepsilon}\right\|_{L^{2}\left(\mathbb{R}^{N}\right)}+\left\|\tau_{r_{\varepsilon} b_{j}}\left(\widetilde{v}_{\varepsilon}\right)-\widetilde{v}_{\varepsilon}\right\|_{L^{2}\left(\mathbb{R}^{N}\right)}
$$

We proceed by getting bounds for each term in (A.3).

Observe that $\mathbb{R}^{N}$ can be represented as the union of the following four disjoint sets:

$$
\begin{array}{ll}
\mathbb{R}^{N} \backslash\left(\Omega_{\varepsilon} \cup \tau_{-\varepsilon b_{j}}\left(\Omega_{\varepsilon}\right)\right) & \text { on which both } \tau_{\varepsilon b_{j}}\left(\widetilde{v}_{\varepsilon}\right) \text { and } \widetilde{v}_{\varepsilon} \text { vanish, } \\
\Omega_{\varepsilon} \cap\left(\mathbb{R}^{N} \backslash \tau_{-\varepsilon b_{j}}\left(\Omega_{\varepsilon}\right)\right) & \text { on which } \tau_{\varepsilon b_{j}}\left(\widetilde{v}_{\varepsilon}\right) \text { vanishes, } \\
\tau_{-\varepsilon b_{j}}\left(\Omega_{\varepsilon}\right) \cap\left(\mathbb{R}^{N} \backslash \Omega_{\varepsilon}\right) & \text { on which } \widetilde{v}_{\varepsilon} \text { vanishes, } \\
\Omega_{\varepsilon} \cap \tau_{-\varepsilon b_{j}}\left(\Omega_{\varepsilon}\right) . &
\end{array}
$$

We now compute $\left\|\tau_{\varepsilon b_{j}}\left(\widetilde{v}_{\varepsilon}\right)-\widetilde{v}_{\varepsilon}\right\|_{L^{2}\left(\mathbb{R}^{N}\right)}^{2}$ on each of these subsets.

First, $\left\|\tau_{\varepsilon b_{j}}\left(\widetilde{v}_{\varepsilon}\right)-\widetilde{v}_{\varepsilon}\right\|_{L^{2}\left(\mathbb{R}^{N} \backslash\left(\Omega_{\varepsilon} \cup \tau_{-\varepsilon b_{j}}\left(\Omega_{\varepsilon}\right)\right)\right.}^{2}=0$.

Next,

and similarly,

$$
\left\|\tau_{\varepsilon b_{j}}\left(\widetilde{v}_{\varepsilon}\right)-\widetilde{v}_{\varepsilon}\right\|_{L^{2}\left(\tau_{-\varepsilon b_{j}}\left(\Omega_{\varepsilon}\right) \cap\left(\mathbb{R}^{N} \backslash \Omega_{\varepsilon}\right)\right)}^{2}=\left\|\tau_{\varepsilon b_{j}}\left(v_{\varepsilon}\right)\right\|_{L^{2}\left(\tau_{-\varepsilon b_{j}}\left(\Omega_{\varepsilon}\right) \cap\left(\mathbb{R}^{N} \backslash \Omega_{\varepsilon}\right)\right)}^{2},
$$

$$
\left\|\tau_{\varepsilon b_{j}}\left(\widetilde{v}_{\varepsilon}\right)-\widetilde{v}_{\varepsilon}\right\|_{L^{2}\left(\Omega_{\varepsilon} \cap\left(\mathbb{R}^{N} \backslash \tau_{-\varepsilon b_{j}}\left(\Omega_{\varepsilon}\right)\right)\right.}^{2}=\left\|v_{\varepsilon}\right\|_{L^{2}\left(\Omega_{\varepsilon} \cap\left(\mathbb{R}^{N} \backslash \tau_{-\varepsilon b_{j}}\left(\Omega_{\varepsilon}\right)\right)\right.}^{2}
$$

These two terms are equal and can be estimated by $2 \|\left. v_{\varepsilon}\right|_{L^{2}\left(D_{\varepsilon}\right)} ^{2}$ where $D_{\varepsilon}$ is the $\varepsilon\left|b_{j}\right|$ neighborhood of $\partial \Omega$. Because the boundary is lipschitz-continuous, this term is bounded above, via the Poincaré inequality, by $\left(c \varepsilon\left|b_{j}\right|\right)^{2}\left\|\nabla v_{\varepsilon}\right\|_{L^{2}\left(D_{\varepsilon}\right)}^{2}$, where $c$ depends on $\partial \Omega$.

To estimate the last term, for $k=\left(k_{i}\right)_{i=1, \ldots, N} \in \mathbb{Z}^{N}$, set $\mathscr{\mathscr { Y }} \varepsilon, k=\varepsilon\left(\mathscr{\mathscr { F }} \varepsilon+\sum_{i=1}^{N} k_{i} b_{i}\right)$ and write

$$
\widetilde{v}_{\varepsilon}=\left.\sum_{k \in \mathbb{Z}^{N}} v_{\varepsilon}\right|_{\mathscr{Y} \varepsilon, k} \chi_{\mathscr{Y} \varepsilon, k}, \quad \tau_{\varepsilon b_{j}}\left(\widetilde{v}_{\varepsilon}\right)=\sum_{k \in \mathbb{Z}^{N}} \tau_{\varepsilon b_{j}}\left(\left.v_{\varepsilon}\right|_{\mathscr{Y} \varepsilon, k}\right) \chi_{\tau_{-\varepsilon b_{j}}(\mathscr{Y} \varepsilon, k}
$$

and

$$
\tau_{\varepsilon b_{j}}\left(\widetilde{v}_{\varepsilon}\right)-\widetilde{v}_{\varepsilon}=\sum_{k \in \mathbb{Z}^{N}}\left(\tau_{\varepsilon b_{j}}\left(\left.v_{\varepsilon}\right|_{\mathscr{Y} \varepsilon, k^{\prime}}\right)-\left.v_{\varepsilon}\right|_{\mathscr{Y} \varepsilon, k}\right) \chi_{\mathscr{Y} \varepsilon, k},
$$

where $\left(k-k^{\prime}\right)_{i}=\left(\delta_{i j}\right)$ for $i=1, \ldots, N$. From Lemma A.1 and by scaling we obtain

$$
\left\|\tau_{\varepsilon b_{j}}\left(\left.v_{\varepsilon}\right|_{\mathscr{H}_{\varepsilon, k^{\prime}}}\right)-\left.v_{\varepsilon}\right|_{\mathscr{Y}_{\varepsilon, k}}\right\|_{L^{2}\left(\mathscr{Y}_{\varepsilon, k}\right)} \leq \sqrt{2} \varepsilon C^{\prime}\left\|\nabla v_{\varepsilon}\right\|_{L^{2}\left(\mathscr{Y}_{\varepsilon, k} \cup \mathscr{Y}_{\varepsilon, k^{\prime}}\right)}
$$


By summing we have

which implies

$$
\left\|\tau_{\varepsilon b_{j}}\left(v_{\varepsilon}\right)-v_{\varepsilon}\right\|_{L^{2}\left(\Omega_{\varepsilon} \cap \tau_{-\varepsilon b_{j}}\left(\Omega_{\varepsilon}\right)\right)} \leq 2 \varepsilon C^{\prime}\left\|\nabla v_{\varepsilon}\right\|_{L^{2}\left(\Omega_{\varepsilon}\right)},
$$

A similar computation shows that

$$
\left\|\tau_{\varepsilon b_{j}}\left(\widetilde{v}_{\varepsilon}\right)-\widetilde{v}_{\varepsilon}\right\|_{L^{2}\left(\mathbb{R}^{N}\right)} \leq 2 \varepsilon\left(C^{\prime}+c\right)\left\|\nabla v_{\varepsilon}\right\|_{L^{2}\left(\Omega_{\varepsilon}\right)} .
$$

$$
\left\|\tau_{r_{\varepsilon} b_{j}}\left(\widetilde{v}_{\varepsilon}\right)-\widetilde{v}_{\varepsilon}\right\|_{L^{2}\left(\mathbb{R}^{N}\right)} \leq 2 \varepsilon\left(C^{\prime}+c\right)\left\|\nabla v_{\varepsilon}\right\|_{L^{2}\left(\Omega_{\varepsilon}\right)} .
$$

Finally, using (A.5) and (A.6) in (A.3) yields

$$
\left\|\tau_{\ell b_{j}}\left(\widetilde{v}_{\varepsilon}\right)-\widetilde{v}_{\varepsilon}\right\|_{L^{2}\left(\mathbb{R}^{N}\right)} \leq 2 \varepsilon\left(1+\left|\kappa_{\varepsilon}\right|\right)\left(C^{\prime}+c\right)\left\|\nabla v_{\varepsilon}\right\|_{L^{2}\left(\mathbb{R}^{N}\right)} .
$$

Letting $\varepsilon$ go to zero, and using the hypotheses on $v_{\varepsilon}$, we get

$$
\left\|\tau_{\ell b_{j}}(v)-v\right\|_{L^{2}\left(\mathbb{R}^{N}\right)} \leq 2\left|l b_{j}\right| C_{1} .
$$

This work is part of the European Research and Training Network "HMS 2000" of the European Union under Contract HPRN-2000-00109. The authors are thankful to H. Wallin for having originally drawn their attention to works of Jones, and to A. Corbo-Esposito for fruitful discussions and references concerning John domains. They also thank D. Cioranescu for some helpful remarks.

\section{REFERENCES}

[1] E. Acerbi, V. Chiado' Piat, G. Dal Maso and D. Percivale, An extension theorem for connected sets, and homogenization in general periodic domains. Nonlinear Anal. TMA 18 (1992) 481-495.

[2] G. Allaire and F. Murat, Homogenization of the Neumann problem with non-isolated holes. Asymptot. Anal. 7 (1993) 81-95.

[3] H. Attouch, Variational convergence for functions and operators. Pitman, Boston, Appl. Math. Ser. (1984).

[4] N.S. Bakhvalov and G.P. Panasenko, Homogenization: Averaging Processes in Periodic Media. Kluwer, Dordrecht (1989).

[5] A. Bensoussan, J.-L. Lions and G. Papanicolaou, Asymptotic Analysis for Periodic Structures. North-Holland, Amsterdam (1978).

[6] B. Bojarski, Remarks on Sobolev imbedding inequalities, in Complex Analysis. Springer-Verlag, Lecture Notes in Math. 1351 (1988) $257-324$.

[7] M. Briane, Poincare'-Wirtinger's inequality for the homogenization in perforated domains. Boll. Un. Mat. Ital. B 11 (1997) 53-82.

[8] M. Briane, A. Damlamian and P. Donato, $H$-convergence in perforated domains, in Nonlinear Partial Differential Equations Appl., Collège de France Seminar, Vol. XIII, edited by D. Cioranescu and J.-L. Lions. Longman, New York, Pitman Res. Notes in Math. Ser. 391 (1998) 62-100.

[9] S. Buckley and P. Koskela, Sobolev-Poincaré implies John. Math. Res. Lett. 2 (1995) 577-593.

[10] D. Chenais, On the existence of a solution in a domain identification problem. J. Math. Anal. Appl. 52 (1975) 189-219.

[11] D. Cioranescu and P. Donato, An Introduction to Homogenization. Oxford University Press, Oxford Lecture Ser. in Math. Appl. 17 (1999).

[12] D. Cioranescu and J. Saint Jean Paulin, Homogenization in open sets with holes. J. Math. Anal. Appl. 71 (1979) $590-607$.

[13] D. Cioranescu and J. Saint Jean Paulin, Homogenization of reticulated structures. Springer-Verlag, Berlin, New York (1999).

[14] C. Conca and P. Donato, Non-homogeneous Neumann problems in domains with small holes. ESAIM: M2AN 22 (1988) 561-608.

[15] A. Damlamian and P. Donato, Homogenization with small shape-varying perforations. SIAM J. Math. Anal. 22 (1991) 639-652.

[16] F.W. Gehring and O. Martio, Quasiextremal distance domains and extension of quasiconformal mappings. J. Anal. Math. 45 (1985) 181-206.

[17] F.W. Gehring and B.G. Osgood, Uniform domains and the quasi-hyperbolic metric. J. Anal. Math. 36 (1979) 50-74.

[18] E. Hruslov, The asymptotic behavior of solutions of the second boundary value problem under fragmentation of the boundary of the domain. Maths. USSR Sbornik 35 (1979).

[19] P. Jones, Quasiconformal mappings and extensions of functions in Sobolev spaces. Acta Math. 1-2 (1981) 71-88.

[20] O. Martio, Definitions for uniform domains. Ann. Acad. Sci. Fenn. Ser. A I Math. 5 (1980) 179-205.

[21] O. Martio, John domains, bilipschitz balls and Poincaré inequality. Rev. Roumaine Math. Pures Appl. 33 (1988) $107-112$. 
[22] O. Martio and J. Sarvas, Injectivity theorems in plane and space. Ann. Acad. Sci. Fenn. Ser. A I Math. 4 (1979) 383-401. [23] V.G. Maz'ja, Sobolev spaces. Springer-Verlag, Berlin (1985).

[24] F. Murat, H-Convergence, Séminaire d'Analyse Fonctionnelle et Numérique (1977/1978). Université d'Alger, Multigraphed.

[25] F. Murat and L. Tartar, H-Convergence, in Topics in the Mathematical Modelling of Composite Materials, edited by A. Cherkaev and R. Kohn. Birkhäuser, Boston (1997) 21-43.

[26] E. Sanchez-Palencia, Non homogeneous Media and Vibration Theory. Springer-Verlag, Lecture Notes in Phys. 127 (1980).

[27] W. Smith and D.A. Stegenga, Hölder domains and Poincaré domains. Trans. Amer. Math. Soc. 319 (1990) 67-100.

[28] S. Spagnolo, Sulla convergenza di soluzioni di equazioni paraboliche ed ellittiche. Ann. Sc. Norm. Sup. Pisa 22 (1968) 571-597.

[29] E.M. Stein, Singular integrals and differentiability properties of functions. Princeton University Press, Princeton, N.J. (1970).

[30] L. Tartar, Cours Peccot au Collège de France (1977).

[31] J. Väisalä, Uniform domains. Tohoku Math. J. 40 (1988) 101-118.

[32] H. Wallin, The trace to the boundary of Sobolev spaces on a snowflake. Manuscripta Math. 73 (1991) 117-125.

[33] V.V. Zhikov, Connectedness and Homogenization. Examples of fractal conductivity. Sbornik Math. 187 (1196) $1109-1147$. 